\title{
Real Dynamics of Integrable Birational Maps*
}

\author{
Anna Cima and Francesc Mañosas \\ Dept. de Matemàtiques, Facultat de Ciències, \\ Universitat Autònoma de Barcelona, \\ 08193 Bellaterra, Barcelona, Spain \\ cima@mat.uab.es, manyosas@mat.uab.es
}

February 26, 2015

\begin{abstract}
We begin by studying the dynamic generated by iteration of birational maps in $\mathbb{R}^{k}$ with $k-1$ independent rational first integrals. We prove that each level curve can be desingularized and compactified being homeomorphic to a finite union of disjoint circles and open intervals. Furthermore, the map can be extended homeomorphically in a natural way to this space. After, we focus our attention in the case that the map has a rational invariant measure and we see that in most cases the orbit of a point or it is periodic or it fulfills densely some connected components of its corresponding level set. Some applications in dimension two and three are presented.
\end{abstract}

2000 Mathematics Subject Classification: 39A11, 39A20.

Keywords: Birational maps, circle maps, difference equations, discrete dynamical systems, first integrals, integrable maps, Lie-symmetries, periodic orbits, rotation numbers.

\section{Introduction}

In this paper we study the dynamic generated by iteration of birational maps $F(x), x \in \mathbb{R}^{k}$ having $k-1$ functionally independent rational first integrals.

A birational map is a map with rational components such that it has an inverse also rational. Recall that a first integral of the dynamical system generated by a map $F$ is a nonconstant $\mathbb{R}$-valued function $H$ which is constant on the orbits of $F$. That is, $H(p)=H(F(p))$ for all $p$ such that the above equality has sense. A set $H_{1}, H_{2}, \ldots, H_{\ell}$ of first integrals of $F$ is said to be functionally independent if the rank of the matrix $(D H)(p)$ is $\ell$ for all $p$ (except perhaps for $p$ belonging to a zero measure set) where $H=\left(H_{1}, H_{2}, \ldots, H_{\ell}\right)$. Clearly,

\footnotetext{
*The authors are partially supported by grants MTM2008-03437, MTM2008-01486 and 2009SGR-410.
} 
the maximum number $\ell$ of functionally independent first integrals for $F$ is $k$. When $\ell=k$ it can be proved that in most cases there exists some $p \in \mathbb{N}$ such that $F^{p}=I d$, see [4]. The case $\ell=k-1$ is sometimes called in the literature, the integrable case. So we are interested in integrable birational maps having rational first integrals. Examples of such a maps are studied in a large number of papers, see for instance [1], [2], [9], [10], [12], [15], $[16],[17],[18],[19]$ and [26] for maps defined in $\mathbb{R}^{2}$ and $[5],[7],[11],[14],[21],[22]$ and [23] for maps defined in $\mathbb{R}^{k}$ with $k>2$.

In general a birational map $F$ is not defined in all $\mathbb{R}^{k}$. In some points $\|F\|$ goes to infinity when we approach to these points. This fact suggests to consider the extension of $F$ on the projective space $P\left(\mathbb{R}^{k}\right)$. But also in this case there are some points of indeterminacy, i. e., points in which $F$ can not be extended in a continuous way. To avoid this problem most of the above cited papers reduce the study on some invariant open regions of $\mathbb{R}^{k}$ in which the birational map $F$ is well defined. The goal of this paper is to determine the behavior of the orbit of every point $x \in \mathbb{R}^{k}$ such that $F^{n}(x)$ is well defined for all $n$. Our approach is the study of the dynamics of $F$ restricted to each invariant level, that in the integrable case is a curve that can have several connected components. Roughly speaking our results say that each level can be compactified and desingularized obtaining a finite union of disjoint circles and intervals. Moreover $F$ can be redefined in this compactified space in such a way that it is a homeomorphism.

Although the results that we get could also be obtained by using results of real algebraic geometry (see [9], [20] and [24] for the planar case), we prefer our approach which mainly uses results and tools of dynamical systems.

To state the main results we need to introduce some notation.

For a birational map $F$ we will denote by $G$ its rational inverse. Assuming $F$ birational integrable on $\mathbb{R}^{k}$, consider $H_{1}, H_{2}, \ldots, H_{k-1}$, a rational family of independent first integrals. Clearly this family is also a rational family of independent first integrals for $G$. Given a first integral $H=\frac{P}{Q}$ with $P$ and $Q$ coprimes and $c \in \mathbb{R}$ the set $\left\{x \in \mathbb{R}^{k}: P(x)=c Q(x)\right\}$ will be called the $c$ level set of $H$ and it will be denoted by the equation $H(x)=c$. We also consider the case $c=\infty$ that corresponds to the set $\left\{x \in \mathbb{R}^{k}: Q(x)=0\right\}$. We denote by $\overline{\mathbb{R}}=\mathbb{R} \cup\{\infty\}$ and for any $\mathbf{c}=\left(c_{1}, \ldots, c_{k-1}\right) \in \overline{\mathbb{R}}^{k-1}$ set

$$
\Sigma_{\mathbf{c}}=\left\{x \in \mathbb{R}^{k}: H_{i}(x)=c_{i}, \text { for } i=1, \ldots k-1\right\} .
$$

For any $\mathbf{c} \in \overline{\mathbb{R}}^{k-1}$ the set $\Sigma_{\mathbf{c}}$ will be called a $\mathbf{c}$ level set of $H_{1}, H_{2}, \ldots, H_{k-1}$. Note that the level sets of $H_{1}, H_{2}, \ldots, H_{k-1}$ are $F$-invariants and also $G$-invariants. Note also that generically these level sets are one dimensional algebraic sets (this is due to the fact that $H_{1}, H_{2}, \ldots, H_{k-1}$ are functionally independents), and hence all the points except perhaps a finite subset (the set of singular points) admit a neighborhood which is homeomorphic to 
an open interval of the real line. However for some values of $\mathbf{c}, \Sigma_{\mathbf{c}}$ could have dimension greater than one as algebraic set. For instance, consider the case where two first integrals $H_{1}$ and $H_{2}$ have the same denominator (this occurs in the 3-dimensional Lyness equation, see Section 3.3). In this setting the equations $H_{1}=\infty$ and $H_{2}=\infty$ are the same equation and hence the level set $\mathbf{c}=\left(\infty, \infty, a_{3}, \ldots, a_{k-1}\right)$ will be defined by at most $k-2$ polynomial equations. Hence its dimension is at least 2. Nevertheless this situation can occur only in a negligible subset of the levels. In fact for a level $\mathbf{c}$ consider $x \in \Sigma_{\mathbf{c}}$ such that the map $H=\left(H_{1}, H_{2}, \ldots, H_{k-1}\right)$ is well defined. Then either the rank of $D(H)(x)$ is $k-1$ and in this case the implicit function theorem implies that $\Sigma_{\mathbf{c}}$ has dimension one locally at $x$, or $D(H)(x)$ has rank less than $k-1$. In this last case Sard's theorem implies that $\mathbf{c}$ belongs to a negligible set in $\mathbb{R}^{k-1}$.

Given a birational map $F$ consider the determinant of the jacobian matrix of $F$ at $x$, which clearly is a rational function. Let $C(F)$ (respectively $D(F)$ ) be the set of points $x \in \mathbb{R}^{k}$ such that $\operatorname{det}(D F)(x)=0$ (respectively $\operatorname{det}(D G)=0$ ). We say that the level set $\Sigma_{\mathbf{c}}$ is regular if it has dimension one and $\Sigma_{\mathbf{c}} \cap C(F)$ and $\Sigma_{\mathbf{c}} \cap D(F)$ are finite sets.

For a one-dimensional algebraic set $X$ we denote by $S(X)$ the set of singular points of $X$. It is known that $S(X)$ is a finite set. In all the paper "almost all $p \in A$ " means for all $p \in A$ except, perhaps, for a finite subset of $A$.

We begin by distinguishing between two algebraic subsets of $\Sigma_{\mathbf{c}}$, being one of them the good set for the study of the iterates of $F$. After, we prove that this good set can be desingularized and compactified in such a way that the study of $F$ reduces to the study of some orientation preserving homeomorphisms on circles and intervals.

Our main result is the following theorem:

Theorem A. Let $F(x), x \in \mathbb{R}^{k}$ be a birational map having $H_{1}, H_{2}, \ldots, H_{k-1}$ rational first integrals functionally independent, let $G(x)$ be its rational inverse, and let $\Sigma_{\mathbf{c}}$ be a regular level set of $H=\left(H_{1}, H_{2}, \ldots, H_{k-1}\right)$. Then

1. $\Sigma_{\mathbf{c}}$ can be decomposed as the union of two algebraic sets $\Sigma_{\mathbf{c}}^{1}$ and $\Sigma_{\mathbf{c}}^{2}$ that do not share irreducible components satisfying the following properties:

(a) For all points $x \in \Sigma_{\mathbf{c}}^{1} \backslash \Sigma_{\mathbf{c}}^{2}$ the birational maps $F$ and $G$ are well defined at $x$. Moreover for these points $F(x), G(x) \in \Sigma_{\mathbf{c}}^{1}$.

(b) For each $x \in \Sigma_{\mathbf{c}}^{2}$ there exists $n$ and $m$ such that $F^{n}$ and $G^{m}$ are not defined at $x$.

2. Let $x \in S\left(\Sigma_{\mathbf{c}}^{1}\right)$ not isolated in $\Sigma_{\mathbf{c}}^{1}$ such that $F^{n}(x)$ is defined for all $n \geq 0$. Then the orbit of $x$ is periodic and contained in $S\left(\Sigma_{\mathbf{c}}^{1}\right)$. Moreover in this case $x$ has not preimages outside $S\left(\Sigma_{\mathbf{c}}^{1}\right)$. 
3. There exists a compactification $\bar{\Sigma}_{\mathbf{c}}^{1}$ of $\Sigma_{\mathbf{c}}^{1} \backslash S\left(\Sigma_{\mathbf{c}}^{1}\right)$ obtained by adding a finite set of points to $\Sigma_{\mathbf{c}}^{1} \backslash S\left(\Sigma_{\mathbf{c}}^{1}\right)$ and an extension $\bar{F}: \bar{\Sigma}_{\mathbf{c}}^{1} \longrightarrow \bar{\Sigma}_{\mathbf{c}}^{1}$ of $F$ such that:

(a) $\bar{\Sigma}_{\mathbf{c}}^{1}$ has a finite number of connected components, which are, topologically, circles and closed intervals:

$$
\bar{\Sigma}_{\mathbf{c}}^{1}=\left(\cup_{i=1}^{s} S_{i}\right) \cup\left(\cup_{j=1}^{r} I_{j}\right) .
$$

(b) $\bar{F}$ is a homeomorphism from $\bar{\Sigma}_{\mathbf{c}}^{1}$ to itself. Thus, for all $i=1, \ldots, s$ (respectively, for all $j=1, \ldots, r$ ) there exists a natural number $m$ such that $S_{i}$ (resp. $I_{j}$ ) is invariant by $\bar{F}^{m}$ and $\bar{F}^{m}$ restricted to $S_{i}$ (resp. $I_{j}$ ) is an orientation preserving homeomorphism.

Observe that all the maps studied in the above mentioned papers belong to the class in which we are interested in, but all of them have an extra property: the existence of (at least) one rational function $\mu$ satisfying:

$$
\mu(F(p))=\operatorname{det}(D F)(p) \cdot \mu(p)
$$

for all $p$ such that $F$ and $\mu$ are well defined.

The existence of a function $\mu$ satisfying condition (1) is related with the existence of an invariant measure of $F$ absolutely continous with respect the Lebesgue measure given by $m=\int \lambda d \mathbf{x}$ where $\lambda=1 / \mu$, and $\lambda$ is the Radon-Nikodym derivative of $m$ with respect to $d \mathbf{x}$ (see [[25], Section 0.4]).

A preliminary result related with these kind of integrable maps, is the next one.

Theorem. (See [6]). Let $F: U \rightarrow U$ be a diffeomorphism having $k-1$ functionally independent first integrals $H_{1}, H_{2}, \ldots, H_{k-1}$, where $U$ is an open connected set $U \subset \mathbb{R}^{k}$ and let $\mu$ be a function satisfying condition (1). Then the following statements hold:

(i) The vector fields

$$
X_{\mu}(p)= \begin{cases}\mu(p)\left(-H_{1, y}, H_{1, x}\right), & \text { if } k=2 ; \\ \mu(p)\left(\nabla H_{1}(p) \times \nabla H_{2}(p) \times \cdots \times \nabla H_{n-1}(p)\right), & \text { if } k>2 .\end{cases}
$$

share with $F$ the same set of functionally independent first integrals.

(ii) The function $\mu$ satisfies condition (1) if and only if the vector field $X_{\mu}$ satisfies

$$
X_{\mu}(F(p))=(D F)(p) \cdot X_{\mu}(p) .
$$

(iii) For each $p \in U$ there is a natural number $m$, such that the integral curve of $X_{\mu}$ passing thought $p, \gamma_{p}$, is invariant by $F^{m}$ and: 
(a) If $\gamma_{p}$ is a critical point of $\dot{x}=X_{\mu}(x)$, then $p$ is a fixed point of $F^{m}$.

(b) If $\gamma_{p}$ is a periodic orbit of $\dot{x}=X_{\mu}(x)$, then $F^{m}$ restricted to $\gamma_{p}$ is conjugated to a rotation of the circle.

(c) If $\gamma_{p}$ is diffeomorphic to the real line then $F^{m}$ restricted to $\gamma_{p}$ is conjugated to a translation of the line.

Some authors say the vector field $X_{\mu}$ in the above theorem is a Lie-symmetry of $F$ (see, for instance, [13]). This theorem cannot be applied directly to our context, because in general, birational mappings are not defined in the whole $\mathbb{R}^{k}$. However we can generalize it to obtain a more precise description of the dynamics in the algebraics sets $\Sigma_{\mathbf{c}}^{1}$. In order to state our result accurately we need to introduce some more notations.

Given an integrable birational map $F$ on $\mathbb{R}^{k}$ with rational first integrals and given a rational function $\mu$ satisfying condition (1), for each $\mathbf{c}$ such that $\Sigma_{\mathbf{c}}$ is a regular level, we denote by

$$
E_{\mathbf{c}}=\left\{x \in \bar{\Sigma}_{\mathbf{c}}^{1}: X_{\mu} \text { is not defined at } x\right\} \cup\left\{x \in \bar{\Sigma}_{\mathbf{c}}^{1}: X_{\mu}(x)=0\right\},
$$

where $\bar{\Sigma}_{\mathbf{c}}^{1}$ is defined and constructed in Theorem A, and $X_{\mu}$ is the vector field defined in Theorem 1. We call $E_{\mathbf{c}}$ the exceptional set of $\Sigma_{\mathbf{c}}$. Notice that $E_{\mathbf{c}}$ contains the points in $\Sigma_{\mathbf{c}}^{1}$ at which the vector field $X_{\mu}$ is not defined as well as the points added to $\Sigma_{\mathbf{c}}^{1}$ to compactify and desingularize it.

On the other hand we say that a fixed point $x$ of a map $f$ is weakly-isolated if for any neighborhood $\mathcal{V}$ of $x$ there exists some $y \in \mathcal{V}$ such that $f(y) \neq y$. We will say that a $n$ periodic point $x$ of $f$ is weakly-isolated if $x$ is a weakly-isolated fixed point of $f^{n}$. Notice that if $x$ is an isolated fixed point in the usual sense, then it is also a weakly-isolated fixed point.

Theorem B. Let $F(x), x \in \mathbb{R}^{k}$ be a birational map having $H_{1}, H_{2}, \ldots, H_{k-1}$ rational first integrals functionally independent and an associated vector field given by (2) with $\mu$ a rational function satisfying condition (1). Let $\Sigma_{\mathbf{c}}$ be a regular level, and let

$$
\bar{\Sigma}_{\mathbf{c}}^{1}=\left(\cup_{i=1}^{s} S_{i}\right) \cup\left(\cup_{j=1}^{r} I_{j}\right)
$$

be the set defined in Theorem A. Assume that $\bar{\Sigma}_{\mathbf{c}}^{1} \cap E_{\mathbf{c}}$ is a finite set. For $i=1, \ldots$ s set $m_{i}$ the smallest natural number such that $\bar{F}^{m_{i}}$ is an orientation preserving homeomorphism of $S_{i}$ and for $j=1, \ldots, r$ set $l_{j}$ the smallest natural number such that $\bar{F}^{l_{j}}$ is an orientation preserving homeomorphism of $I_{j}$. Then the following assertions hold:

(a) $\left.\bar{F}^{m_{i}}\right|_{S_{i}}$ is either conjugated to a rotation or there is some weakly-isolated periodic orbit contained in $E_{\mathbf{c}} \cap S_{i}$. In this last case all weakly-isolated orbits in $S_{i}$ are contained in 
$E_{\mathbf{c}}$, the rotation number is rational, and the alpha and omega limit of all non-periodic points are some of these weakly-isolated orbits.

(b) $\left.\bar{F}^{l_{j}}\right|_{I_{j}}$ is either the identity or it has some weakly-isolated fixed point $x$. In this case $x \in E_{\mathbf{c}}$ and the alpha and omega limit of all non-fixed points are some of these weaklyisolated fixed points.

In the view of the result established in Theorem B, we give the following definition:

Definition 1. We say that the dynamic of a point $p$ is standard if either, the orbit of $p$ is periodic or it fulfills densely some connected components of its corresponding level set.

We notice that if the exceptional set $E_{\mathbf{c}}$ does not contain finite invariant subsets, then the dynamics of any point of $\Sigma_{\mathbf{c}}^{1}$ is standard.

In order to do a description of the dynamic of a concrete birational integrable map with rational first integrals and with a rational function $\mu(p)$ satisfying condition (1), it is necessary to study the topology of its level curves and apply Theorems A and B. In Section 3 we give some examples in order to show how to apply the general results. Specifically, we study the two and the three dimensional Lyness equation and a family of area preserving mappings.

Finally we propose the following problem:

Open problem. Given a birational integrable map with rational first integrals, does always exists a rational function $\mu(p)$ satisfying condition (1), and therefore a rational Liesymmetry?

In all the exemples that we have found in the literature the above question has an affirmative answer. On the other hand we point out that if the map is integrable but not rational then there are examples with a negative answer to the problem, see [8].

The paper is organized as follows. In Section 2 we prove Theorems A and B while in Section 3 some examples and applications are given.

Acknowledgements We want to thank Joaquim Roé for his advising in the results of Real Algebraic Geometry and also to Armengol Gasull for stimulating and helpful indications.

\section{Proofs of Theorems A and B}

First of all we recall some notations and results on real algebraic geometry which we freely will use trough the paper. An algebraic set $X$ on $\mathbb{R}^{k}$ is the set of common real zeros of a finite set of polynomials $p_{1}, \ldots, p_{s} \in \mathbb{R}\left[x_{1}, \ldots, x_{k}\right]$. As usual we call $X$ the variety of $p_{1}, \ldots, p_{s}$ and we will write $X=V\left(p_{1}, \ldots, p_{s}\right)$. Clearly $V\left(p_{1}, \ldots, p_{s}\right)=V\left(<p_{i}, \ldots, p_{s}>\right)$ 
where $<p_{1}, \ldots, p_{s}>$ denotes the ideal generated by $p_{1}, \ldots, p_{s}$. We say that an algebraic set $X=V(I)$ is irreducible if $X=Y \cup Z$ with $Y$ and $Z$ algebraic sets, implies that either $X=Y$ or $X=Z$. It is well known that this occurs if and only if $\operatorname{rad}(I)$ is a prime real ideal (see [3]). It is also well known that any algebraic set $X$ decomposes as a finite union of irreducible algebraic sets called the irreducible components of $X$.

We will say that an algebraic set $X$ has dimension 0 if it is a finite set. We will say that an irreducible algebraic set $X$ has dimension 1 if it is not a finite set of points and if any algebraic set $Y \varsubsetneqq X$ has dimension 0 . We will say that an algebraic set has dimension 1 if all its irreducible components have dimension 1 or 0 and at least one of them has dimension 1.

Let $X$ be a one-dimensional algebraic set. It is well known that there exists a minimal finite subset of $X, S$ such that $X \backslash S$ is a differentiable sub-manifold of $\mathbb{R}^{k}$. The elements of $S$ are called the singular points of $X$.

Next proposition is the first step to prove Theorem A.

Proposition 2. Let $F: \mathbb{R}^{k} \longrightarrow \mathbb{R}^{k}$ be a birational map having $H_{1}, H_{2}, \ldots, H_{k-1}$ rational first integrals functionally independent, and let $\Sigma_{\mathbf{c}}$ be a regular level set of $H=$ $\left(H_{1}, H_{2}, \ldots, H_{k-1}\right)$. Then $\Sigma_{\mathbf{c}}$ can be decomposed as the union of two algebraic sets $\Sigma_{\mathbf{c}}^{1}$ and $\Sigma_{\mathbf{c}}^{2}$, that do not share irreducible components, satisfying the following properties:

(a) For each $x \in \Sigma_{\mathbf{c}}^{2}$ there exists $n$ and $m$ such that $F^{n}$ and $G^{m}$ are not defined at $x$.

(b) The set $\Sigma_{\mathbf{c}}^{1} \cap \Sigma_{\mathbf{c}}^{2}$ is a finite set.

(c) For all points $x \in \Sigma_{\mathbf{c}}^{1} \backslash \Sigma_{\mathbf{c}}^{2}$ the birational maps $F$ and $G$ are well defined at $x$. Moreover for these points $F(x), G(x) \in \Sigma_{\mathbf{c}}^{1}$.

Proof.

Denote by $A$ (respectively $B$ ) the set of points where $F$ (respectively $G$ ) is not defined. Clearly $A$ and $B$ are algebraic sets. Let $M$ be a irreducible one dimensional component of $\Sigma_{\mathbf{c}}$. Thus $A \cap M \subset M$ is an algebraic set and since $M$ is irreducible it follows that either $A \cap M=M$ or $A \cap M$ is a finite set of points. Thus, given an irreducible component $M$ either $F$ is not defined at each point of $M$ or $F$ is defined in almost all points of $M$. The same situation holds for $G$. We denote by $F^{-1}(M)$ the set $F^{-1}(M)=\left\{x \in \Sigma_{\mathbf{c}}: F(x) \in M\right\}$. Note that $F^{-1}(M)=X \backslash A$ where $X$ is a one dimensional algebraic set contained in $\Sigma_{\mathbf{c}}$. Note also that each irreducible component of $X$ either is contained in $A$ or cuts $A$ in a finite set. It implies that either $F^{-1}(M)$ is empty or adding a finite set of points to $F^{-1}(M)$ (in fact, the closure of $F^{-1}(M)$ in the Zariski topology) we get an algebraic set. In this last case we still denote by $F^{-1}(M)$ the algebraic set obtained adding these points. Clearly the same situation holds for $G$. 
Let $M$ be an irreducible one-dimensional component of $\Sigma_{\mathbf{c}}$ and assume that $F$ is defined on $M$. We claim that in the same sense as before (that is perhaps adding a finite set of points) $F(M)$ is an algebraic set having only one irreducible one dimensional component of $\Sigma_{\mathbf{c}}$. Since $F$ is defined in $M$ and $\Sigma_{\mathbf{c}}$ is regular it follows that $F$ is defined at $x$ and $\operatorname{det}(D F)(x) \neq 0$ for almost all $x \in M$. Then from the inverse function theorem we get that $G$ is defined at $F(x)$ and $F^{-1}(F(x))=G(F(x))=x$ for almost all $x \in M$. Set $x \in M$, not isolated in $M$ and such that $F$ is defined at $x$. Also from the regularity of $\Sigma_{\mathbf{c}}$ it follows that $F(x)$ is not isolated on its level set and hence it belongs to some one-dimensional component of the level. Then a neighborhood $V$ of $x$ in $M$ has the property that $F(V)$ cuts at least one dimensional irreducible component of $\Sigma_{\mathbf{c}}$ in one arc. Denoting this component by $N$ we claim that $N \subset F(M)$. Note that from the previous observation $G$ is defined on $N$ and $G^{-1}(M)$ is a one dimensional algebraic set which cuts $N$ in one arc. Since $N$ is irreducible then $N \subset G^{-1}(M)$ and also from the above observation it follows that $N \subset F(M)$. This proves that $F(M)$ is an algebraic set. Now to finish the claim it remains to prove that $F(M)$ has only one irreducible one dimensional component. Assume that $F(M)$ has more than one irreducible one-dimensional components, say $N_{1}, \ldots, N_{k}$. Then $G\left(N_{i}\right)=M$ for $i=1, \ldots, k$. This implies that almost all points on $M$ have more than one preimage by $G$ which implies that $\operatorname{det}(D G)=0$ in these preimages which contradicts the regularity of $\Sigma_{\mathbf{c}}$. Thus the claim follows. Note also that the same argument proves that $F^{-1}(M)$ has at most one dimensional irreducible component when $M$ is irreducible.

Recall that $\Sigma_{\mathbf{c}} \cap A$ is an algebraic set. Assume that it has some one dimensional irreducible component $M$. We claim that there exists $k \geq 0$ such that $F^{-k}(M)$ is a finite set of points. If $F^{-1}(M)$ is not a finite set, then from the above observation it is an irreducible component of $\Sigma_{\mathbf{c}}$. Since the number of irreducible components is finite it follows that either there exits $k$ such that $F^{-k}(M)$ is a finite set or there exists $r<s$ such that $F^{-r}(M)$ and $F^{-s}(M)$ are irreducible one dimensional components and $F^{-r}(M)=F^{-s}(M)$. In the second case, it follows that $M=F^{r-s}(M)$. This is a contradiction, because by construction $F$ is defined at $F^{r-s}(M)$, and $M \subset A$. Thus the claim is proved.

Notice that if $k$ is the first natural number such that $F^{-k}(M)$ is finite then for almost all $y \in F^{-k+1}(M), G$ is not defined at $y$.

Let $M_{1}, \ldots, M_{n}$ be the set of irreducible components of $\Sigma_{\mathbf{c}} \cap A$. For $j=1, \ldots, n$ let $i_{j}$ be the largest natural number such that $F^{-i_{j}}\left(M_{j}\right)$ is not a finite set. We define

$$
\Sigma_{\mathbf{c}}^{2}=\cup_{j=1}^{n}\left(\cup_{l=0}^{i_{j}} F^{-l}\left(M_{j}\right)\right) .
$$

By construction $\Sigma_{\mathbf{c}}^{2}$ satisfies $(a)$.

Let us denote by $\mathcal{B}$ the set of irreducible components of $\Sigma_{\mathbf{c}}$ not contained in $\Sigma_{\mathbf{c}}^{2}$. Then 
we define

$$
\Sigma_{\mathbf{c}}^{1}=\cup_{B_{i} \in \mathcal{B}} B_{i} .
$$

Clearly $\Sigma_{\mathbf{c}}^{1}$ is an algebraic set and $\Sigma_{\mathbf{c}}=\Sigma_{\mathbf{c}}^{1} \cup \Sigma_{\mathbf{c}}^{2}$. Since by construction both algebraic sets do not share irreducible components, statement (b) follows. This implies that $F$ is defined for all $x \in \Sigma_{\mathbf{c}}^{1}$ except for the points in $\Sigma_{\mathbf{c}}^{1} \cap \Sigma_{\mathbf{c}}^{2}$.

Set $B_{l} \in \mathcal{B}$. We have that $F\left(B_{l}\right)$ is also a 1-dimensional irreducible component. If $F\left(B_{l}\right) \subset \Sigma_{\mathbf{c}}^{2}$ then by construction $B_{l} \subset \Sigma_{\mathbf{c}}^{2}$ which gives a contradiction. Thus $F\left(B_{l}\right) \in \mathcal{B}$. So $F$ acts on $\mathcal{B}$. Moreover this action is injective, because if $F\left(B_{i}\right)=F\left(B_{j}\right)$ then $J F$ is identically 0 on $B_{i} \cup B_{j}$ which contradicts the hypotheses.

To finish the proof it suffices to show that $G$ also acts on $\mathcal{B}$. Suppose to arrive a contradiction that $G$ is not defined at $B \in \mathcal{B}$. Then since $B_{1}=F(B) \in \mathcal{B}$ it follows that $G^{-1}(B)=B_{1}$. Iterating this argument and since $F$ acts injectively on $\mathcal{B}$ we will obtain that there exists $k$ be such that $G^{-k}(B)=B$. But this implies that $G$ is defined on $B$ which gives a contradiction. This ends the proof of the proposition.

In order to illustrate how the decomposition established in Proposition 2 works, we consider the three dimensional Lyness equation.

Example 1. Consider the three dimensional Lyness equation

$$
F(x, y, z)=\left(y, z, \frac{a+y+z}{x}\right),
$$

which is a birational map of $\mathbb{R}^{3}$, having as a rational inverse

$$
G(x, y, z)=\left(\frac{a+x+y}{z}, x, y\right) .
$$

Its first integrals are given by:

$$
\begin{aligned}
& H_{1}(x, y, z)=\frac{(x+1)(y+1)(z+1)(a+x+y+z)}{x y z}, \\
& H_{2}(x, y, z)=\frac{(1+x+y)(1+y+z)(a+x+y+z+x z)}{x y z} .
\end{aligned}
$$

We notice that the level $(\infty, \infty)$ has dimension two and that the level $(0,0)$ contains the line $\{(x, 1-a,-1), x \in \mathbb{R}\}$ where $\operatorname{det}(D F)=0$ and also the line $\{(-1,1-a, z), z \in$ $\mathbb{R}\}$ where $\operatorname{det}(D G)=0$. Some simple computations imply that for all $\mathbf{c}=\left(c_{1}, c_{2}\right)$ with $\left(c_{1}, c_{2}\right) \neq(\infty, \infty)$ and $\left(c_{1}, c_{2}\right) \neq(0,0), \Sigma_{\mathbf{c}}$ is a regular level.

Fix a level $\mathbf{c}=\left(c_{1}, c_{2}\right)$ which is defined trough

$$
\begin{aligned}
& (x+1)(y+1)(z+1)(a+x+y+z)-c_{1} x y z=0, \\
& (1+x+y)(1+y+z)(a+x+y+z+x z)-c_{2} x y z=0 .
\end{aligned}
$$

The map $F$ is not defined when $x=0$. This implies that $(y+1)(z+1)(a+y+z)=0$ and $(1+y)(1+y+z)(a+y+z)=0$. We get the two one-dimensional components:

$$
M_{1}=\{(0,-1, z): z \in \mathbb{R}\} \quad \text { and } \quad M_{2}=\{(0, y,-a-y): y \in \mathbb{R}\} .
$$


The preimages of $M_{1}$ are:

$$
\begin{aligned}
& F^{-1}\left(M_{1}\right)=\{(\lambda, 0,-1): \lambda \in \mathbb{R}, \lambda \neq 0\}, \\
& F^{-2}\left(M_{1}\right)=\{(-(a+\lambda), \lambda, 0): \lambda \in \mathbb{R}, \lambda \neq 0\},
\end{aligned}
$$

and in general $F^{-3}\left(M_{1}\right)$ has a finite number of points in $\Sigma_{\mathbf{c}}$ : these are the points $(x,-(a+$ $\lambda), \lambda)$ with $x \neq 0$ and $\lambda \neq 0$ which satisfy $H_{1}=c_{1}$ and $H_{2}=c_{2}$. Only when $\lambda+1=0$, $F^{-3}\left(M_{1}\right)$ has a continuum of points: the set $\{(x, 1-a,-1)\}$ which is contained in $H_{1}=$ $0, H_{2}=0$. The preimages of $M_{2}$ are:

$$
\begin{aligned}
& F^{-1}\left(M_{2}\right)=\{(-1,0, y): y \in \mathbb{R}\}, \\
& F^{-2}\left(M_{2}\right)=\{(\lambda,-1,0): \lambda \in \mathbb{R}, \lambda \neq 0\},
\end{aligned}
$$

and the only point in $F^{-2}\left(M_{2}\right)$ which has preimage is when $\lambda=1-a$. In this case all the straight line $(x, 1-a,-1)$ collapses to $(1-a,-1,0)$ via $F$. If $x \neq 0$ then $H_{1}(x, 1-a,-1)=$ $0=H_{2}(x, 1-a,-1)$, while the point $(0,1-a,-1) \in \Sigma_{\mathbf{c}}$ for all regular level c. Hence, in general, $F^{-3}\left(M_{2}\right)$ reduces to the point $(0,1-a,-1)$. So, in this example,

$$
\Sigma_{\mathbf{c}}^{2}=\left(\cup_{l=0}^{2} F^{-l}\left(M_{1}\right)\right) \cup\left(\cup_{l=0}^{2} F^{-l}\left(M_{2}\right)\right),
$$

for all regular level $\mathbf{c}$.

In the case that $\mathbf{c}=(0,0)$ some simple computations show that the preimages of the set in which $F$ is not defined run all the set $\Sigma_{(0,0)}$, that is, the orbit of the points in $\Sigma_{(0,0)}$ is not entirely defined.

Notice that from the above Proposition we see that if $\Sigma_{\mathbf{c}}$ is a regular level then the dynamics of $F$ and $G$ are concentrate on the algebraic set $\Sigma_{\mathbf{c}}^{1}$. However there is a finite subset of $\Sigma_{\mathbf{c}}^{1}$ in which $F$ or $G$ are not defined.

Our next step is to extend $F$ and $G$ to these points. Recall that since $\Sigma_{\mathbf{c}}^{1}$ is a one dimensional algebraic set it has only a finite number of singularities.

Lemma 3. Let $\Sigma_{\mathbf{c}}$ be a regular level and let $x$ be a non-singular point of $\Sigma_{\mathbf{c}}^{1}$. Assume that $F$ is not defined at $x$. Then either

$$
\lim _{y \rightarrow x, y \in \Sigma_{\mathbf{c}}^{1}}\|F(y)\|=\infty
$$

or

$$
\lim _{y \rightarrow x, y \in \Sigma_{\mathbf{c}}^{1}} F(y)
$$

exists and $F$ can be extended analytically at $x$. 
Proof. Since $x$ is a non-singular point of $\Sigma_{\mathbf{c}}$ it follows that there exist neighborhoods $U$ of $x$ in $\Sigma_{\mathbf{c}}^{1}$ and $I$ of $0 \in \mathbb{R}$ and an analytic map $\varphi: I \longrightarrow U$ such that $\varphi(0)=x$ and $\varphi^{\prime}(0) \neq 0$. Let $F_{i}, i=1, \ldots k$ to denote the components of $F$. Thus

$$
\lim _{y \rightarrow x, y \in \Sigma_{\mathbf{c}}^{1}} F_{i}(y)=\lim _{t \rightarrow 0} F_{i}(\varphi(t)) .
$$

The result follows from the fact that $F_{i}(\varphi(t))$ is a meromorphic function in one variable.

From now on we denote again by $F$ the extension of $F$ that ensures the above lemma. So at this point we can assume that if $F$ is not defined at $x \in \Sigma_{\mathbf{c}}^{1}$ then either $\lim _{y \rightarrow x, y \in \Sigma_{\mathbf{c}}^{1}}\|F(y)\|=$ $\infty$ or $x$ is a singular point. Clearly the same situation holds for $G$. Next lemma shows that when $F$ is defined then it is a local homeomorphism.

Lemma 4. Let $\Sigma_{\mathbf{c}}$ be a regular level set and let $x \in \Sigma_{\mathbf{c}}^{1}$. Assume that $F$ is defined at $x$ and $x$ and $F(x)$ are non singular points in $\Sigma_{\mathbf{c}}^{1}$. Then there exists an open neighborhood $U$ of $x$ in $\Sigma_{\mathbf{c}}$ such that $F$ maps homeomorphically $U$ on its image.

Proof. Since $F(x)$ is non singular we can consider a small neighborhood $W$ of $F(x)$ in $\Sigma_{\mathbf{c}}^{1}$ homeomorphic to an open interval. Since $F$ is continuous at $x$ there exists $U$, a neighborhood of $x$ in $\Sigma_{\mathbf{c}}$, homeomorphic to an open interval, such that $F(U) \subset W$. If $\left.F\right|_{U}$ is not injective then either, there is a subinterval of $U$ in which $F$ is constant or there is a subinterval of $W$ which is covered two times by $\left.F\right|_{U}$. In the first case $U$ contains an interval in which the $\operatorname{det}(D F)$ is zero. In the second one we get the same situation for $W$ and the $\operatorname{det}(D G)$. Both conclusions contradict the properties of $\Sigma_{\mathbf{c}}^{1}$.

The next step is to desingularize and compactify $\Sigma_{\mathbf{c}}^{1}$. With this aim we denote by $S\left(\Sigma_{\mathbf{c}}^{1}\right)$ the set of singular points of $\Sigma_{\mathbf{c}}^{1}$.

Consider $\Sigma_{\mathbf{c}}^{1} \backslash S\left(\Sigma_{\mathbf{c}}^{1}\right)$. This set can be described as

$$
\Sigma_{\mathbf{c}}^{1} \backslash S\left(\Sigma_{\mathbf{c}}^{1}\right)=\left(\cup_{i=1}^{r} I_{i}\right) \cup\left(\cup_{i=1}^{s} S_{i}\right),
$$

where each $I_{i}$ is homeomorphic to an open interval of the line and each $S_{i}$ is homeomorphic to a circle. We consider $r$ disjoint open intervals $J_{i}=\left(a_{i}, b_{i}\right)$ in $\mathbb{R}$ without common boundaries with an homeomorphism $\varphi_{i}$ from $J_{i}$ to $I_{i}$ for each $i=1,2, \ldots, k-1$. Set

$$
\Omega_{\mathbf{c}}=\left(\cup_{i=1}^{r} J_{i}\right) \cup\left(\cup_{i=1}^{s} S_{i}\right)
$$

and

$$
\varphi: \Omega_{\mathbf{c}} \longrightarrow \Sigma_{\mathbf{c}}^{1} \backslash S\left(\Sigma_{\mathbf{c}}^{1}\right)
$$

defined by 


$$
\varphi(x)= \begin{cases}\varphi_{i}(x), & \text { if } x \in J_{i} \text { for some } i \\ x, & \text { otherwise. }\end{cases}
$$

Clearly $\varphi$ is a homeomorphism between $\Omega_{\mathbf{c}}$ and $\Sigma_{\mathbf{c}}^{1} \backslash S\left(\Sigma_{\mathbf{c}}^{1}\right)$. Let $\tilde{F}=\varphi^{-1} \circ F \circ \varphi$ and $\tilde{G}=\varphi^{-1} \circ G \circ \varphi$ be the natural versions of $F$ and $G$ in $\Omega_{\mathbf{c}}$. Since $F$ (respectively $F^{-1}$ ) and $\tilde{F}$ (respectively $\tilde{F}^{-1}$ ) are conjugated we fix our attention to the dynamics of $\tilde{F}$ and $\tilde{F}^{-1}$. Note that $\tilde{F}$ and $\tilde{F}^{-1}$ are continuous and monotonic in the points where they are defined.

Set $\bar{J}_{i}=\left[a_{i}, b_{i}\right]$ and $\bar{\Omega}_{\mathbf{c}}=\left(\cup_{i=1}^{r} \bar{J}_{i}\right) \cup\left(\cup_{i=1}^{s} S_{i}\right)$. Then the boundary of $\bar{\Omega}_{\mathbf{c}}, \partial\left(\Omega_{\mathbf{c}}\right)=$ $\cup_{i=1}^{r}\left\{a_{i}, b_{i}\right\}$.

Definition 5. We say that a point $x \in \Sigma_{\mathbf{c}}^{1} \backslash S\left(\Sigma_{\mathbf{c}}^{1}\right)$ is a fork point for $F$ if either, its image under $F$ is a singular point of $\Sigma_{\mathbf{c}}^{1}$ or $\lim _{y \rightarrow x, y \in \Sigma_{\mathbf{c}}^{1}}\|F(y)\|=\infty$. If $x$ is a fork point for $F$ we will say that $\varphi^{-1}(x)$ is a fork point for $\tilde{F}$.

Let $x \in \Sigma_{\mathbf{c}}^{1} \backslash S\left(\Sigma_{\mathbf{c}}^{1}\right)$ be a fork point for $F$. If $F(x)$ is a singular point then $\tilde{F}$ is not defined at $\varphi^{-1}(x)$. However $\lim _{y \rightarrow \varphi^{-1}(x)^{+}}(\tilde{F}(y))$ and $\lim _{y \rightarrow \varphi^{-1}(x)^{-}}(\tilde{F}(y))$ exist, and both belong to $\partial\left(\Omega_{\mathbf{c}}\right)$. We point out that these limits are distinct. Otherwise some points on the left and on the right side of $\varphi^{-1}(x)$ have the same image under $\tilde{F}$. This implies that there are infinitely many points near $F(x)$ in which $G$ is not defined, a contradiction with the regular character of $\Sigma_{\mathbf{c}}$. Clearly the same situation occurs for $\varphi^{-1}(x)$ when $\lim _{y \rightarrow x, y \in \Sigma_{\mathbf{c}}^{1}}\|F(y)\|=\infty$. Then the only possible continuous extension of $\tilde{F}$ at the fork points, gives us a bivaluated "function".

Also when $x \in \partial\left(\Omega_{\mathbf{c}}\right)$ the map $\tilde{F}$ can be easily extended to $x$ through $\tilde{F}(x)=\lim _{y \rightarrow x} \tilde{F}(y)$. This limit exists by compacity and monotonicity arguments. Note that if $x \in \partial\left(\Omega_{\mathbf{c}}\right)$ verifies that $\tilde{F}(x) \notin \partial\left(\Omega_{\mathbf{c}}\right)$ then $\tilde{F}(x)$ is a fork point for $\tilde{G}$.

In short we have extended $\tilde{F}$ to $\bar{\Omega}_{\mathbf{c}}$ with the pathology that it is bivaluated at the fork points. Moreover in the non-fork points it is continuous and monotone. We have to do some identifications in order to obtain a well defined function. First, we prove the following lemma.

Lemma 6. Given a fork point $x$ of $\tilde{F}$ there exists a finite number of pairs $\left(P_{i}, Q_{i}\right), i=$ $1,2, \ldots, l$ with $P_{i}, Q_{i} \in \partial \Omega_{\mathbf{c}}$ and a fork point $y$ of $\tilde{G}$ such that the following scheme works:

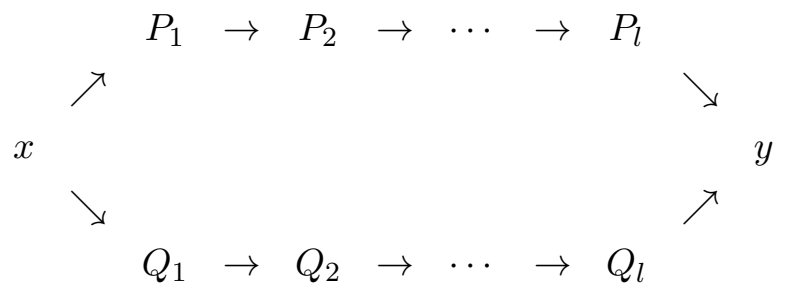

where the arrows mean images under $\tilde{F}$. Furthermore all the points $P_{i}, Q_{i}$ which appear in (5) are distinct. 
Proof of Lemma 6. Take $x$ a fork point and let $P_{1}, Q_{1}$ be the two lateral limits of $\tilde{F}(y)$ when $y \rightarrow x$. First of all we claim that there exits $n \in \mathbb{N}$ such that $\tilde{F}^{n}\left(P_{1}\right) \notin \partial\left(\Omega_{\mathbf{c}}\right)$. If not, since $\partial\left(\Omega_{\mathbf{c}}\right)$ is finite, in the sequence $\tilde{F}^{n}\left(P_{1}\right)$ there are some repetitions. Let $i>0$ the first natural number such that $\tilde{F}^{i}\left(P_{1}\right) \in\left\{P_{1}, \tilde{F}\left(P_{1}\right), \ldots, \tilde{F}^{i-1}\left(P_{1}\right)\right\}$ and let $0 \leq j<i$ be such that $\tilde{F}^{j}\left(P_{1}\right)=\tilde{F}^{i}\left(P_{1}\right)$. If $\tilde{F}^{i}\left(P_{1}\right) \neq P_{1}$ we get that $j \geq 1$ and then $\tilde{F}\left(\tilde{F}^{j-1}\left(P_{1}\right)\right)=\tilde{F}\left(\tilde{F}^{i-1}\left(P_{1}\right)\right)$. Note that by construction $\tilde{F}^{j-1}\left(P_{1}\right) \neq \tilde{F}^{i-1}\left(P_{1}\right)$. This fact implies that points near $\tilde{F}^{j-1}\left(P_{1}\right)$ have the same image that points near $\tilde{F}^{i-1}\left(P_{1}\right)$. Translating this behavior to $\Sigma_{\mathbf{c}}$ this implies that there are infinitely many points in which $G$ is not defined contradicting our assumption that $\Sigma_{\mathbf{c}}$ is regular. If $\tilde{F}^{i}\left(P_{1}\right)=P_{1}$ we get the same situation related to points near $P_{1}$ and points in one side of $x$. Thus the claim follows.

Let $n$ be the first natural number such that $\tilde{F}^{n}\left(P_{1}\right)=y \notin \partial\left(\Omega_{\mathbf{c}}\right)$. Now we claim that $\tilde{F}^{n}\left(Q_{1}\right)=y$ and for all $0 \leq i<n, \tilde{F}^{i}\left(Q_{1}\right) \in \partial\left(\Omega_{\mathbf{c}}\right)$. First of all note that since for all $0 \leq i<n, \tilde{F}^{i}\left(P_{1}\right) \in \partial\left(\Omega_{\mathbf{c}}\right)$, we get that $x$ is a fork point for $\tilde{F}^{i+1}$. This implies that $\tilde{F}^{i}\left(Q_{1}\right) \in \partial\left(\Omega_{\mathbf{c}}\right)$. On the other hand since one lateral limit of $\tilde{F}^{n+1}$ at $x$ is $y \notin \partial\left(\Omega_{\mathbf{c}}\right)$ this implies that the other lateral limit also is equal to $y$. Lastly the fact that all the boundary points appearing in this scheme are different follows again from the fact that $\Sigma_{\mathbf{c}}$ is regular.

Definition 7. Given $P, Q \in \partial\left(\Omega_{\mathbf{c}}\right)$, we say that $P \sim Q$ if and only if either, $P=Q$ or it exists a fork point $x$ and a natural number $k$ such that $\tilde{F}^{k}(x)=\{P, Q\}$.

Lemma 8. The relation $P \sim Q$ defined above in $\partial\left(\Omega_{\mathbf{c}}\right)$ is an equivalence relation. Each equivalence class has at most two points.

Proof Assume that $P \sim Q$ and $Q \sim R$, i. e., $\tilde{F}^{k}(x)=\{P, Q\}$ and $\tilde{F}^{s}(y)=\{Q, R\}$ for certain $k, s \in \mathbb{N}$ and $x, y$ fork points. From Lemma 6 , using the local injectivity of $F$ and $G$, it follows that $k=s, x=y$ and consequently $P=R$.

Let $\bar{\Sigma}_{\mathbf{c}}^{1}$ be the set $\overline{\Omega_{\mathbf{c}}}$ with the above identifications. We notice that since the points of $\Omega_{\mathbf{c}}$ are unique in its equivalence class we can consider $\Omega_{\mathbf{c}}$ as a subset of $\bar{\Sigma}_{\mathbf{c}}^{1}$.

Proposition 9. With the above notations the following hold:

(1) $\bar{\Sigma}_{\mathbf{c}}^{1}=\left(\cup_{i=1}^{l} K_{i}\right) \cup\left(\cup_{i=1}^{m} S_{i}\right)$ where $K_{i}, i=1, \ldots l$ are disjoint closed intervals and $S_{i}, i=1, \ldots, m$ are disjoint topological circles. The connected components of $\bar{\Sigma}_{\mathbf{c}}^{1}$ are precisely $K_{1}, \ldots, K_{l}, S_{1} \ldots, S_{m}$.

(2) The map $\tilde{F}$ can be extended to a homeomorphism $\bar{F}$ on $\bar{\Sigma}_{\mathbf{c}}^{1}$ and for each connected component $M$ of $\bar{\Sigma}_{\mathbf{c}}$ there exists a natural number $k$ such that $\bar{F}^{k}$ is an orientation preserving map from $M$ to itself. 
Proof. Statement (1) follows from the fact that $\overline{\Omega_{\mathbf{c}}}=\left(\cup_{i=1}^{r} J_{i}\right) \cup\left(\cup_{i=1}^{s} S_{i}\right)$ and we have identified some of the boundary points of the intervals $J_{i}$. Since each boundary point can be identified at most with another boundary point it follows that, with this procedure, we only can obtain intervals or circles.

Now we prove (2). We begin by proving that $\bar{F}$ is well defined and injective on $\bar{\Sigma}_{\mathbf{c}}^{1}$.

Assume that $x \in \bar{\Sigma}_{\mathbf{c}}^{1}$ is not a fork point for $\tilde{F}$. Then $\bar{F}(x)=\tilde{F}(x)$ and $\bar{F}$ is injective in this set.

Let $x$ be a fork point and consider its corresponding scheme (10). Then $\bar{F}(x)=P_{1}=Q_{1}$. Since $P_{i}=Q_{i}$ for $i=1,2, \ldots, l$ we see that $\bar{F}$ is compatible with the identifications. We notice that if $x, y$ is a pair of distinct fork points, then $\bar{F}(x) \neq \bar{F}(y)$.

Now assume that $P \in \partial\left(\Omega_{\mathbf{c}}\right)$ and that the equivalence class of $P$ has two different elements $P$ and $Q$. Then, $P, Q$ are contained in a scheme like (10), so they are identified and $\bar{F}(P)=\bar{F}(Q)$. If two of such points have the same image, then necessarily they are in the same equivalence class and so, they coincide.

Finally if a point $P \in \partial\left(\Omega_{\mathbf{c}}\right)$ is the only one in its equivalence class, then we define $\bar{F}(P)=\lim _{x \rightarrow P} \tilde{F}(x)$. In this case $\bar{F}(P) \in \partial\left(\Omega_{\mathbf{c}}\right)$ and also $\bar{F}(P)$ is the only one in its equivalence class. So $\bar{F}$ just gives a permutation on the set of these points. Moreover no different points of this type can have the same image under $\bar{F}$.

Until now we have seen that $\bar{F}$ is well defined and injective on $\bar{\Sigma}_{\mathbf{c}}^{1}$. For the same reason the map $\bar{G}$ is well defined and injective on $\bar{\Sigma}_{\mathbf{c}}^{1}$. And of course, $\bar{F}$ (and $\bar{G}$ ) are continuous in $\bar{\Sigma}_{\mathbf{c}}^{1}$. Since $\bar{F}$ is an homeomorphism it must be a permutation in the set of connected components. This ends the proof of the proposition.

Proof of Theorem A. Statements (1) and (3) follow from Proposition 2 and Proposition 9. Now we prove statement (2). Assume that $x \in S\left(\Sigma_{\mathbf{c}}^{1}\right)$ is not isolated in $\Sigma_{\mathbf{c}}^{1}$. Let $l$ be the number of branches of $\Sigma_{\mathbf{c}}^{1}$ at $x$, that is the number of connected components of $\left(\Sigma_{\mathbf{c}}^{1} \backslash\{x\}\right) \cap V$ where $V$ is a small neighborhood of $x$ in $R^{k}$. Clearly $l>2$. If $F(x)=x$ then the orbit of $x$ is periodic. Assume that $F(x) \neq x$. Since $\Sigma_{c}$ is a regular level then the number of branches at $F(x)$ is greater or equal to $l$. Hence $F(x)$ is a singular point. Iterating this argument it follows that $F^{i}(x)$ is a singular point for all $i \geq 0$. Denoting by $l_{i}$ the number of branches at $F^{i}(x)$ it also follows that $l_{i} \leq l_{j}$ when $i<j$. Since the number of singularities is finite it follows that there exists a first pair $i<j$ such that $F^{i}(x)=F^{j}(x)$ and from the above observation $l_{i}=l_{m}=l_{j}$ for all $m$ between $i$ and $j$. We claim that $i$ must be 0 . If not $F^{i}(x)$ has two different preimages $F^{i-1}(x)$ and $F^{j-1}(x)$. Since $l_{i}=l_{j-1}$ and $l_{i-1}>2$ the result follows from the regularity of the level. So the claim is proved and the orbit of $x$ is a periodic orbit of singular points. Furthermore since $x$ has a singular preimage with the same number of branches, by the same argument as before, $x$ can not have a regular preimage. This ends 
the proof of Theorem A.

In order to prove Theorem B, we consider the vector field $X_{\mu}$ defined in Theorem 1 , where the function $\mu$ satisfies the equation 1 , i. e.:

$$
\mu(F(p))=\operatorname{det}(D F)(p) \cdot \mu(p)
$$

for all $p$ such that $F$ and $\mu$ are well defined. Then, the vector field $X_{\mu}$ satisfies:

$$
X_{\mu}(F(p))=(D F)(p) \cdot X_{\mu}(p),
$$

(see [6] for a proof). Let $\varphi(t, p)$ be the solution of $\dot{x}=X_{\mu}(x)$ such that $\varphi(0, p)=p$ defined in its maximal interval $I_{p}$. Taking derivatives in respect to $t$ and using condition (7) we easily get

$$
F\left(\varphi(t, p)=\varphi(t, F(p)), \forall t \in I_{p} \cap I_{F(p)} .\right.
$$

\section{Proof of Theorem B.}

(a) First assume that $E_{c} \cap S_{i}=\emptyset$. Then $S_{i}$ is a periodic orbit of the vector field $X_{\mu}$. Fix $p \in S_{i}$ and let $\tau$ be the less positive real number such that $\varphi(\tau, p)=\bar{F}^{m_{i}}(p)$. Then, from (8) we get that

$$
\bar{F}^{m_{i}}(\varphi(t, x))=\varphi\left(t, \bar{F}^{m_{i}}(x)\right)
$$

and hence $\varphi(\tau, x)=\bar{F}^{m_{i}}(x)$ for any $x \in S_{i}$. Thus $\bar{F}^{m_{i}}$ is conjugated to the rigid rotation $R\left(e^{i t}\right)=e^{i(t+2 \pi \tau / T)}$ where $T$ denotes the period of $S_{i}$ as a periodic orbit of the vector field and being $H\left(e^{i t}\right)=\varphi\left(\frac{T}{2 \pi} t, p\right)$ the conjugation.

Now assume that $E_{\mathbf{c}} \cap S_{i} \neq \emptyset$ and let $y \in E_{\mathbf{c}} \cap S_{i}$. In the case that $\bar{F}^{m_{i}}(y) \notin E_{\mathbf{c}}$, although $X_{\mu}$ is not defined at $y$, we claim that we can extend the flow $\varphi$ at the point $y$, in such a way that condition (9) remains true.

To this end, for any $t \in I_{\bar{F}^{m}} m_{(y)}$ we define

$$
\phi(t, y)=\bar{F}^{-m_{i}}\left(\varphi\left(t, \bar{F}^{m_{i}}(y)\right) .\right.
$$

Let $K$ and $L$ be the two connected components of $S_{i} \backslash E_{\mathbf{c}}$ having $y$ in its closure. Now we are going to extend the flow $\varphi$ at any point $z \in K \cup L$. Set $z \in K$, and consider $I_{z}=\left(t_{z}^{-}, t_{z}^{+}\right)$, the interval of definition of $\varphi(t, z)$. Then we have that $\left.\lim _{t \rightarrow t^{*}} \varphi(t, z)\right)=y$ where $t^{*}$ is one of the endpoints of $I_{z}$. Assume for example that $t^{*}=t_{z}^{+}$. Now we claim that $t_{z}^{+}<\infty$. Clearly it suffices to show that the right endpoint of $I_{w}$ is finite for some $w \in K$. Since $E_{\mathbf{c}} \cap S_{i}$ is a finite set, we can choose $w \in K$ such that $\bar{F}^{m_{i}}(w) \notin E_{\mathbf{c}}$. Furthermore we choose $w$ near $y$ in such a way that $\bar{F}^{m_{i}}(y)$ and $\bar{F}^{m_{i}}(w)$ are in the same connected component of $S_{i} \backslash E_{\mathbf{c}}$. Then if $t^{\prime}$ is such that $\varphi\left(t^{\prime}, \bar{F}^{m_{i}}(y)\right)=\bar{F}^{m_{i}}(w)$, it is clear that $t^{\prime}=t_{w}^{+}$and hence, $t_{w}^{+}$is finite. 
Now for any $t \in\left(t_{z}^{-}, t_{z}^{+}+t_{\bar{F}^{m_{i}(y)}}^{+}\right)$we define

$$
\phi(t, z)= \begin{cases}\varphi(t, z) & \text { if } t \in I_{z} \\ y, & \text { if } t=t_{z}^{+} \\ \bar{F}^{-m_{i}} \varphi\left(t-t_{z}^{+}, \bar{F}^{m_{i}}(y)\right) & \text { if } t>t_{z}^{+} .\end{cases}
$$

This new map $\phi$ is an extension of the previous $\varphi$ and by construction it has the standard properties of the flows and it satisfies condition (9). Iterating this procedure we can extend the map $\varphi$ to all the points in $E_{\mathbf{c}}$ except perhaps to a finite invariant set. If the map can be extended to all $S_{i}$ then arguing as in the previous case we obtain that $\bar{F}^{m_{i}}$ is conjugated to a rotation. If not this implies the existence of a finite invariant subset of $E_{\mathbf{c}}$, which we denote by $\mathcal{P}$. In this case the rotation number of $\bar{F}^{m_{i}}$ restricted to $S_{i}$ is rational and all the points of $\mathcal{P}$ are periodic points with the same period. If we denote by $r$ its period we get that $\bar{F}^{r m_{i}}(p)=p$ for all $p \in \mathcal{P}$. Although in this situation the map $\bar{F}^{r m_{i}}$ could be the identity map and $\bar{F}^{m_{i}}$ would be conjugated to a rotation. But, if it is not the case, there is a weakly-isolated fixed point for $\bar{F}^{r m_{i}}$.

To end the proof of statement $(a)$ it remains to prove that all the weakly-isolated orbits are contained in $E_{\mathbf{c}}$. But it is an easy consequence of condition (9): if $p \notin E_{\mathbf{c}}$, then the equality $\bar{F}^{m_{i}}(\varphi(t, p))=\varphi\left(t, \bar{F}^{m_{i}}(p)\right)=\varphi(t, p)$ implies that $\varphi(t, p)$ is a fixed point for any $t \in I_{p}$, so $p$ is not a weakly-isolated point of $\bar{F}^{r m_{i}}$.

(b) In the case of the interval we point out that by construction $\bar{I}_{j} \cap E_{\mathbf{c}} \neq \emptyset$. Set $J_{j}=\bar{I}_{j} \backslash E_{\mathbf{c}}$. Clearly $J_{j}$ is a finite union of disjoint open intervals. If $\bar{F}^{l_{j}}$ is not the identity map, then it exists some fixed point $x$ of $\bar{F}^{l_{j}}$ which is weakly-isolated. But as in the previous case, condition (8) let us to deduce that if $x \notin E_{\mathbf{c}}$ then $x$ is not a weakly-isolated fixed point.

\section{$3 \quad$ Examples}

\subsection{The map $F(x, y)=\left(y,-x+(2 y) /\left(a^{2}-y^{2}\right)\right)$.}

The area-preserving rational family of mappings

$$
F(x, y)=\left(y,-x-\frac{\beta y^{2}+\epsilon y+\xi}{\alpha y^{2}+\beta y+\gamma}\right)
$$

preserves the foliation

$$
V(x, y)=\alpha x^{2} y^{2}+\beta\left(x^{2} y+x y^{2}\right)+\gamma\left(x^{2}+y^{2}\right)+\epsilon x y+\xi(x+y)=c,
$$

and the dynamic of $F$ is studied in some regions of $\mathbb{R}^{2}$ and for some values of the parameters in different papers (see [2], [12], [17],[18],[19], for instance). 
The method established in the above sections make possible to know the full dynamic of the map for all the values of the parameters. To avoid too many cases, we restrict our attention to the uniparametric family

$$
F(x, y)=\left(y,-x+\frac{2 y}{a^{2}-y^{2}}\right) \quad, \quad \text { with } \quad a>1
$$

which has the first integral

$$
V(x, y)=x^{2} y^{2}-a^{2}\left(x^{2}+y^{2}\right)+2 x y \text {. }
$$

In order to implement our compactification let us introduce the following points, each one of them having the natural meaning:

$$
\begin{array}{rrrrr}
P_{1}=(a,-\infty) & P_{2}=(-\infty,-a) \quad, \quad P_{3}=(-a, \infty) & , \quad P_{4}=(-\infty, a) \\
Q_{1}=(a, \infty) & , \quad Q_{2}=(\infty,-a) \quad, \quad Q_{3}=(-a,-\infty) & , & Q_{4}=(\infty, a) .
\end{array}
$$

Furthermore note that when $y^{2}=a^{2}$ the function $F$ is not defined. Also when $x^{2}=a^{2}$, the formal inverse of $F$,

$$
G(x, y)=\left(-y+\frac{2 x}{a^{2}-x^{2}}, x\right)
$$

is not defined. For all $c$ we consider the four points in $\Sigma_{\mathbf{c}}$ :

$$
\mathbf{x}_{\mathbf{c}}=\left(\frac{c+a^{4}}{2 a}, a\right), \mathbf{y}_{\mathbf{c}}=\left(-a,-\frac{c+a^{4}}{2 a}\right), \mathbf{z}_{\mathbf{c}}=\left(-\frac{c+a^{4}}{2 a},-a\right), \mathbf{t}_{\mathbf{c}}=\left(a, \frac{c+a^{4}}{2 a}\right) .
$$

Lemma 10. With the notations introduced above, the following schemes work:

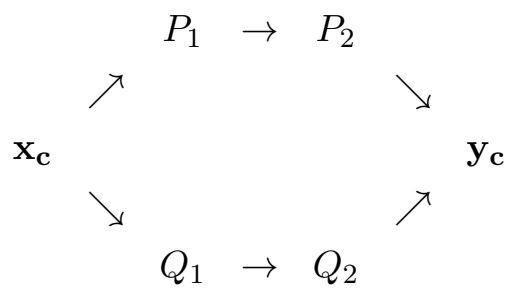

and

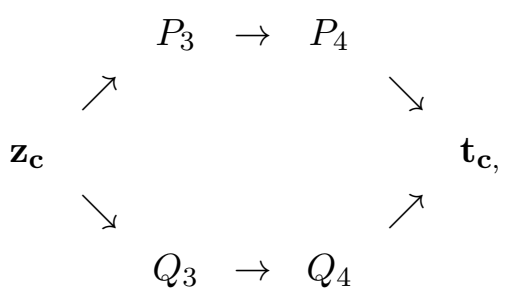

where the arrows mean images under $\bar{F}$.

The proof of Lemma 10 follows straightforward just taking limits on $\Sigma_{\mathbf{c}}$.

From the above lemma we see that for each $c \in \mathbb{R}$, we have to do the identifications: $P_{i} \sim Q_{i}$ for $i=1,2,3,4$. 
On the other hand we notice that since the family of maps $F$ are preserving measure, i.e., $\operatorname{det}(D F)(p)=1$ for all $p$, then any constant function $\mu$ satisfies condition (1) and we can apply Theorem B. To this end we can consider the vector field $X:=\left(-V_{y}, V_{x}\right)$ which has the following expression:

$$
X(x, y)=\left(-2 x^{2} y+2 a^{2} y-2 x\right) \frac{\partial}{\partial x}+\left(2 x y^{2}-2 a^{2} x+2 y\right) \frac{\partial}{\partial y} .
$$

Its critical points are:

$$
\begin{aligned}
& R_{ \pm}:=\left( \pm \sqrt{1+a^{2}}, \mp \sqrt{1+a^{2}}\right) \\
& S_{ \pm}:=\left( \pm \sqrt{a^{2}-1}, \pm \sqrt{a^{2}-1}\right), \\
& O:=(0,0),
\end{aligned}
$$

which correspond to the levels:

$$
\begin{aligned}
& c_{1}:=-\left(a^{2}+1\right)^{2}, \\
& c_{2}:=-\left(a^{2}-1\right)^{2}, \\
& c_{3}:=0 .
\end{aligned}
$$

We point out that the points $\left\{R_{+}, R_{-}\right\}$form a two-periodic orbit of $F$ while $S_{+}, S_{-}$and $O$ are fixed points of $F$.

From now on we write $\alpha(p)$ (respectively, $\omega(p)$ ) to denote the $\alpha$ (respectively, $\omega$ ) limit set of the point $p$.

Theorem 11. Assume that $a>1$ and let $p$ be a point such that $F^{n}(p)$ is defined for all $n \in \mathbb{Z}$. Then,

(1) For all $c \neq c_{1}, c_{2}$ the dynamic of $p$ is standard.

(2) For $p \in \Sigma_{c_{1}}, \alpha(p)=\omega(p)=\left\{R_{+}, R_{-}\right\}$.

(3) For $p \in \Sigma_{c_{2}}$ either, $\omega(p)=S_{+}$and $\alpha(p)=S_{-}$or viceversa.

Proof. For $c \neq c_{1}, c_{2}$ and with the above notations, the set $E_{c}$ reduces to $E_{c}=\left\{P_{1}, P_{2}, P_{3}, P_{4}\right\}$ (where we have identified $P_{i}$ with $Q_{i}$ for $\left.i=1,2,3,4\right)$ and the set $\bar{\Sigma}_{\mathbf{c}}^{1}$ is a finite union of circles. Notice that the point $O$ is isolated in the level curve $\Sigma_{0}$. Therefore in spite of $O$ is a critical point of the differential equation, the set $E_{0}$ does not contain $O$. From Lemma 10 the extended orbit of each one of $P_{i}$ is not periodic. Hence, from Theorem B we know that for each circle $S$ of $\bar{\Sigma}_{\mathbf{c}}^{1}$ there exists a natural number $m$ such that $S$ is invariant for $\bar{F}^{m}$ and $\bar{F}^{m}$ restricted to $S$ is conjugated to a rotation of the circle. So, statement (1) follows.

For $c=c_{1}$ the following equality holds:

$$
V(x, y)-c_{1}=\left(a x+x y+a y+1+a^{2}\right)\left(-a x+x y-a y+1+a^{2}\right) .
$$


Let us call $H_{1}:=\left(a x+x y+a y+1+a^{2}\right)$ and $H_{2}:=\left(-a x+x y-a y+1+a^{2}\right)$. In the intersection of $H_{1}=0$ and $H_{2}=0$ we find the singular points $R_{ \pm}$.

The curve $H_{1}=0$ (respectively $H_{2}=0$ ) is compactified by adding $P_{2}, P_{3}, Q_{2}, Q_{3}$ (respectively $\left.P_{1}, P_{4}, Q_{1}, Q_{4}\right)$ and it contains $\mathbf{x}_{\mathbf{c}}, \mathbf{t}_{\mathbf{c}}$ (respectively $\mathbf{y}_{\mathbf{c}}, \mathbf{z}_{\mathbf{c}}$.)

Simple calculations show that $F$ sends the hyperbola $H_{1}=0$ to $H_{2}=0$ and that $F\left(R_{+}\right)=R_{-}, F\left(R_{-}\right)=R_{+}$. Hence the points $R_{ \pm}$are two periodic by $F$.

Following the procedure explained in Section 2, each singular point $R_{+}$and $R_{-}$must be substituted by four points. Set $M_{i}$ and $N_{i}, i=1,2,3,4$ for the end points of $\bar{\Sigma}_{\mathbf{c}}$, where $M_{1}, M_{3}$ (respectively $N_{2}, N_{4}$ ) are the limits on $H_{1}=0$ and $M_{2}, M_{4}$ (respectively $N_{1}, N_{3}$ ) are the limits on $H_{2}=0$. From Lemma 10 it can be seen that $\bar{F}\left(M_{i}\right)=N_{i}$ and $\bar{F}\left(N_{i}\right)=M_{i}$ for $i=1,2,3,4$.

Calling $I_{1}:=\left[M_{1}, N_{2}\right], I_{2}:=\left[M_{2}, N_{1}\right], I_{3}:=\left[M_{3}, N_{4}\right]$ and $I_{4}:=\left[M_{4}, N_{3}\right]$ we get that $\bar{\Sigma}_{\mathbf{c}}^{1}$ is the union of these four intervals. Each interval $I_{i}$ is invariant by $\bar{F}^{2}$ and the action of $\bar{F}^{2}$ is conjugated to a homeomorphism of the interval with the two boundary points fixed and no other fixed points. So, one of the points is an attractor point and the other one is a repellor point for $\bar{F}^{2}$.

Then the exceptional set $E_{c_{1}}$ contains four isolated two-periodic orbits which are the blow-up of the original two-periodic points $R_{+}, R_{-}$. So, statement (2) follows.

The proof of the last case follows in a similar way. The basic difference is that each interval appearing in the decomposition is invariant by the map.

In order to illustrate better the procedure explained in Section 2 and since the sets $V(x, y)=c$ can easily be drawn, we can do a more accurate description of the dynamics of $F$ on $\Sigma_{\mathbf{c}}$. This description is synthesized in Figure 1 (case $a>1$ ) which we are going to explain when $c<c_{1}=-\left(1+a^{2}\right)^{2}$, see (a) of Figure 1 .

From Lemma 10 and taking into account the identifications $P_{i} \sim Q_{i}$ for $i=1,2,3,4$ we see that $\bar{\Sigma}_{\mathbf{c}}^{1}$ is the union of two circles, one of them containing $P_{1}, P_{3}$ and $\mathbf{y}_{\mathbf{c}}, \mathbf{t}_{\mathbf{c}}$ and the other one containing $P_{2}, P_{4}$ and $\mathbf{x}_{\mathbf{c}}, \mathbf{z}_{\mathbf{c}}$. Again from Lemma 10 , we can see that each circle is invariant by $F^{2}$, and that $F^{2}$ is conjugated to a rotation of the circle. Each one of the circles either, it is densely covered by the orbits of $F^{2}$ when the rotation number is irrational or it is full of periodic points of $F^{2}$.

\subsection{The map $\mathbf{F}(\mathbf{x}, \mathbf{y})=(\mathbf{y},(\mathbf{a}+\mathbf{y}) / \mathbf{x})$.}

The dynamic of the $k$-dimensional Lyness equation $F\left(x_{1}, x_{2}, \ldots, x_{k}\right)=\left(x_{2}, x_{3}, \ldots,(a+\right.$ $\left.\left.\sum_{i=2}^{k} x_{i}\right) / x_{1}\right)$ has been studied in several papers, specially the 2 and 3 dimensional cases and mainly when the parameter $a$ and the initial conditions are positive. In this section we 
describe de behavior of all the orbits of the two dimensional case for all the values of the parameter $a$. We recall that when $a=1$, the above map is globally periodic of period five.

It is well know that it has the first integral

$$
H(x, y)=\frac{(x+1)(y+1)(a+x+y)}{x y} .
$$

The vector filed $X=-\mu(x, y) \frac{\partial}{\partial y} H(x, y)+\mu(x, y) \frac{\partial}{\partial x} H(x, y)$, with $\mu(x, y)=x y$ satisfies $(7)$. 

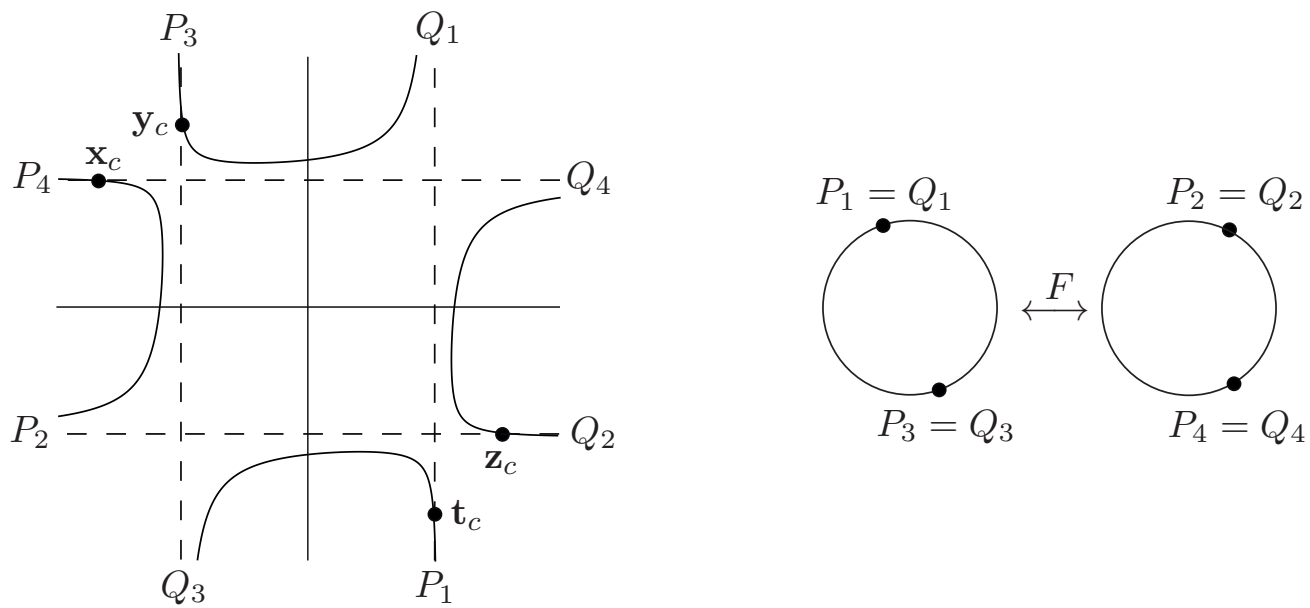

(a) $c<-\left(1+a^{2}\right)^{2}$

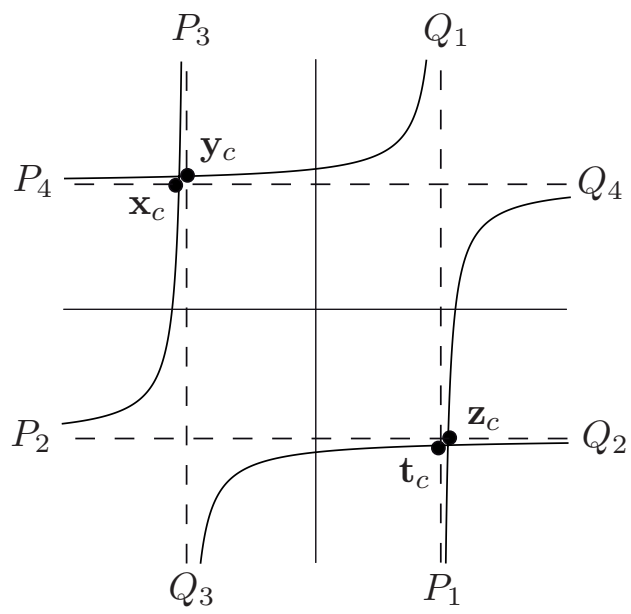

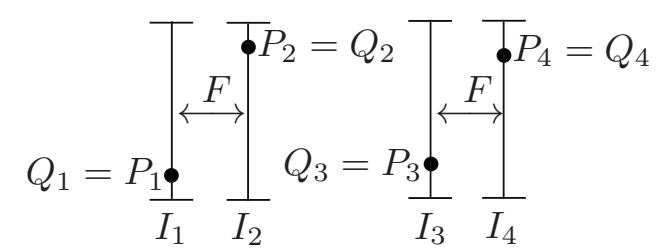

(b) $c=-\left(1+a^{2}\right)^{2}$

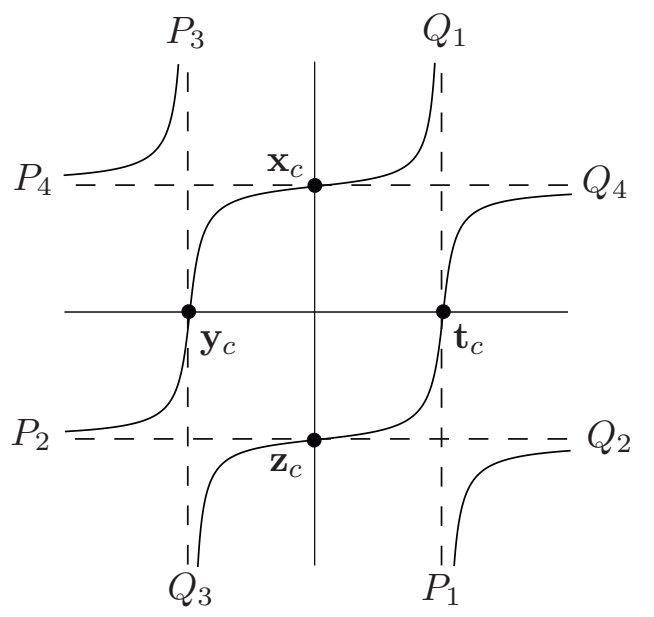

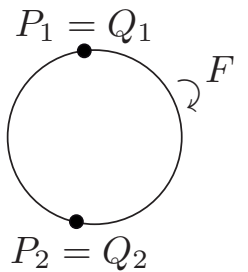

(c) $-\left(1+a^{2}\right)^{2}<c<-\left(a^{2}-1\right)^{2}$ 


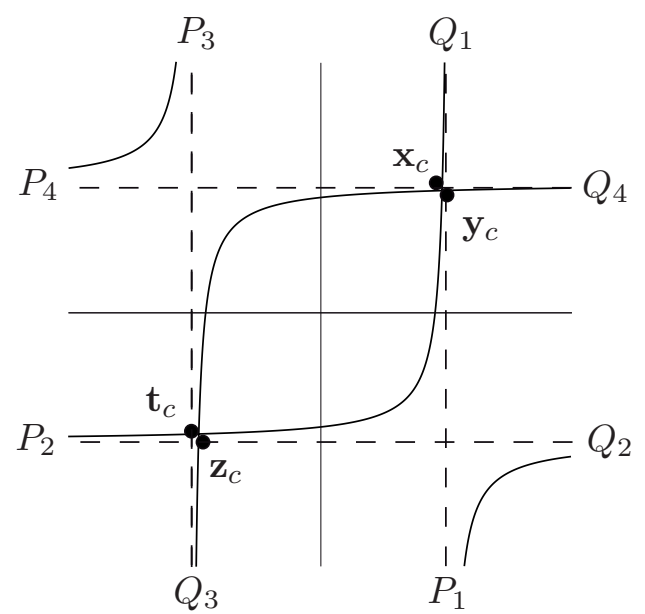

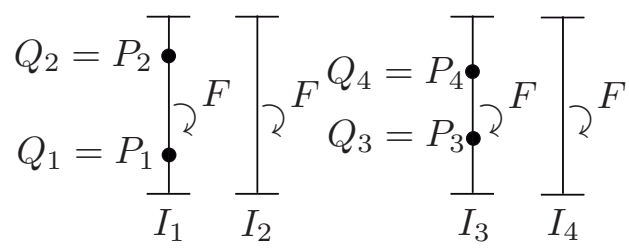

(d) $c=-\left(a^{2}-1\right)^{2}$
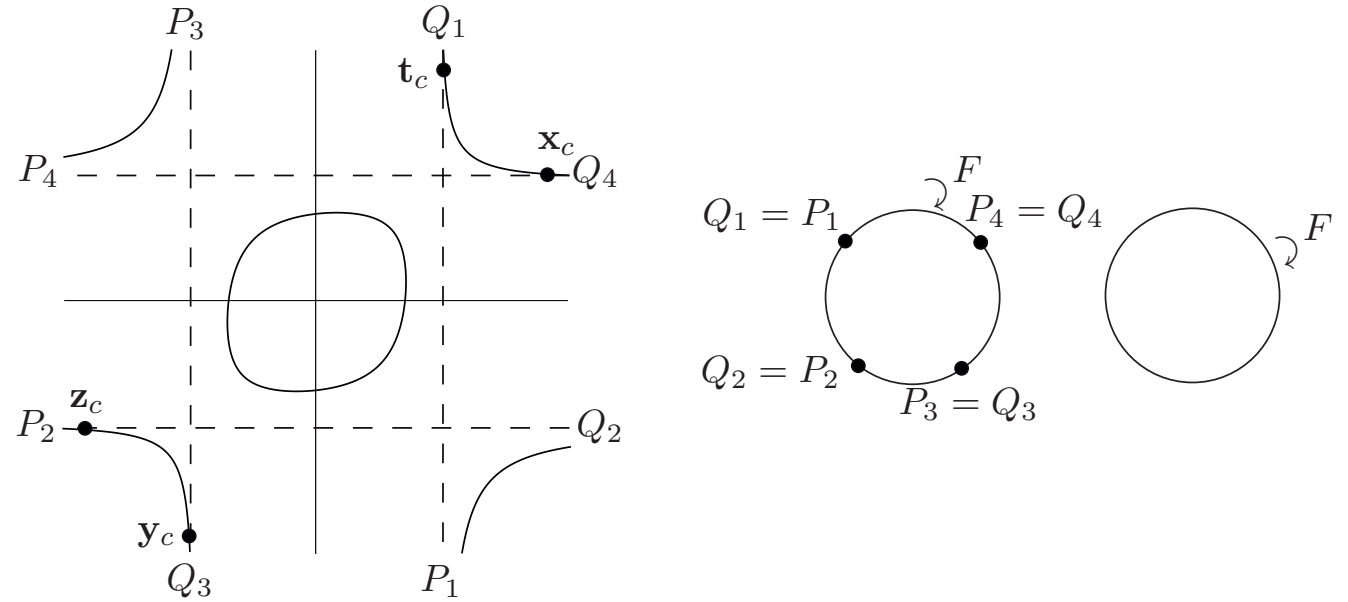

(e) $-\left(a^{2}-1\right)^{2}<c<0$

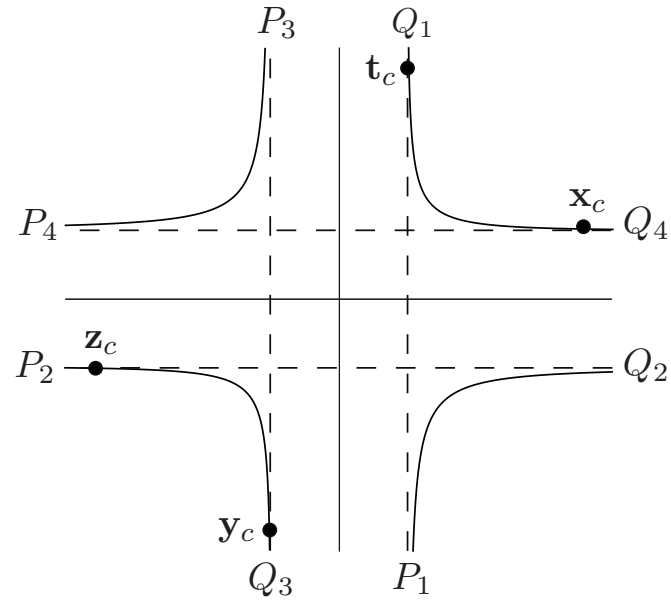

(f) $c>0$

Figure 1: The level curves and its compactification for the map $F(x, y)=\left(y,-x,+\frac{2 y}{a^{2}-y^{2}}\right)$ with $a>1$. 
The critical points, when they are defined, are

$$
\begin{aligned}
O_{1} & =\{(-1,-1),(1-a,-1),(-1,1-a)\}, \\
O_{2} & =\left\{\left(\frac{1+\sqrt{1+4 a}}{2}, \frac{1+\sqrt{1+4 a}}{2}\right)\right\}, \\
O_{3} & =\left\{\left(\frac{1-\sqrt{1+4 a}}{2}, \frac{1-\sqrt{1+4 a}}{2}\right)\right\}, \\
O_{4} & =\left\{\left(\frac{-1 \pm \sqrt{4 a-3}}{2}, \frac{-1 \mp \sqrt{4 a-3}}{2}\right)\right\},
\end{aligned}
$$

which belong to the levels:

$$
\begin{aligned}
& c_{1}=0, \\
& c_{2}=\frac{(3+\sqrt{1+4 a})^{2}(1+a+\sqrt{1+4 a})}{(1+\sqrt{1+4 a})^{2}}, \\
& c_{3}=\frac{(\sqrt{1+4 a}-3)^{2}(1+a-\sqrt{1+4 a})}{(\sqrt{1+4 a}-1)^{2}}, \\
& c 4=a-1,
\end{aligned}
$$

respectively. The points of $O_{2} \cup O_{3}$ are fixed points of $F(x, y)$ while the points of $O_{1}$ (resp. $\mathrm{O}_{4}$ ) form a three (resp. two) periodic orbit of $F(x, y)$.

The results that we obtain are summarized in the next theorem. Its proof follows in a similar way that the proof of Theorem 11 and we omit the details for shortness.

Theorem 12. Assume that $a \neq 1$ and consider a point $p \in \Sigma_{c}$ such that $F^{n}(p)$ is defined for all $n \in \mathbb{Z}$. Then the following hold:

(1) Assume that $a=-1 / 4$. Then, if $c \notin\{0,27 / 4\}$ then the dynamic of $p$ is standard and if $c=0($ resp. $c=27 / 4)$ then $\alpha(p)=\omega(p)=O_{1}$, (resp. $\left.\alpha(p)=\omega(p)=O_{2}=O_{3}\right)$.

(2) Assume that $a<3 / 4, a \neq-1 / 4$. Then, if $c \neq 0$ then the dynamic of $p$ is standard and if $c=0$ then $\alpha(p)=\omega(p)=O_{1}$.

(3) Assume that $a=3 / 4$. Then, if $c \notin\{0,-1 / 4\}$ then the dynamic of $p$ is standard. If $c=0($ resp. $c=-1 / 4)$ then $\alpha(p)=\omega(p)=O_{1}\left(\right.$ resp. $\left.\alpha(p)=\omega(p)=O_{3}=O_{4}\right)$.

(4) Assume that $a>3 / 4$. Then, if $c \neq\{0, a-1\}$ then the dynamic of $p$ is standard and if $c=0$ (resp. $c=a-1)$ then $\alpha(p)=\omega(p)=O_{1},\left(\right.$ resp. $\left.\alpha(p)=\omega(p)=O_{4}\right)$.

\subsection{The map $\mathrm{F}(\mathrm{x}, \mathrm{y}, \mathrm{z})=(\mathrm{y}, \mathrm{z},(\mathrm{a}+\mathrm{y}+\mathrm{z}) / \mathrm{x})$.}

In this section we describe the dynamic of the Lyness equation for $k=3$ considered in the whole $\mathbb{R}^{3}$.

As we noticed in Example 1, the map $F(x, y, z)=\left(y, z, \frac{a+y+z}{x}\right)$ has the two independent first integrals:

$$
\begin{aligned}
& H_{1}(x, y, z)=\frac{(x+1)(y+1)(z+1)(a+x+y+z)}{x y z} \\
& H_{2}(x, y, z)=\frac{(1+x+y)(1+y+z)(a+x+y+z+x z)}{x y z} .
\end{aligned}
$$


On the other hand, the function

$$
\mu(x, y, z)=x(x+1) z(z+1)-(a+x+y+z) y(y+1)
$$

satisfies condition (1), so we can apply Theorem B. The corresponding differential equation is

$$
\begin{aligned}
X(x, y, z) & =\left[x(x+1)(1+y+z)(a+x+y-y z) \frac{\partial}{\partial x}+y(y+1)(x-z)(a+x+y+z+x z) \frac{\partial}{\partial y}\right. \\
& \left.+z(z+1)(1+x+y)(a+y+z-x y) \frac{\partial}{\partial z}\right] /(x y z)
\end{aligned}
$$

In order to state the results we need to introduce the following subsets of $\mathbb{R}^{3}$.

We denote by $\alpha_{1}, \alpha_{2}$ and $\alpha_{3}$ the curves

$$
\begin{aligned}
& \alpha_{1}=\{(-1, t,-1): t \in \mathbb{R} \backslash\{0,1-a\}\} \\
& \alpha_{2}=\{(t,-1-t, t): t \in \mathbb{R} \backslash\{0,-1,1-a\}\} \\
& \alpha_{3}=\left\{\left\{\left(t, \frac{a+t}{t-1}, t\right): t \in \mathbb{R} \backslash\{0,1,-a\} \text { and } P(t) \leq 0\right\}\right.
\end{aligned},
$$

where $P(t)=(t+1)\left(2 t^{2}+(3 a-1) t+1-a\right)(2 t+a-1)\left(t^{2}+t+a-1\right)^{2}$.

Simple computations show that the points in $\alpha_{1}$ are 4-periodic for $F$ and $F^{2}\left(\alpha_{1}\right) \subset \alpha_{1}$. The points in $\alpha_{2}$ are 6-periodic for $F$ and $F^{3}\left(\alpha_{2}\right) \subset \alpha_{2}$. Lastly points in $\alpha_{3}$ are 2-periodic for $F$. Thus we also define the following invariant sets:

$$
\begin{aligned}
\gamma_{1} & =\alpha_{1} \cup F\left(\alpha_{1}\right), \\
\gamma_{2} & =\alpha_{2} \cup F\left(\alpha_{2}\right) \cup F^{2}\left(\alpha_{2}\right), \\
\gamma_{3} & =\alpha_{3} \cup F\left(\alpha_{3}\right) .
\end{aligned}
$$

And we denote by $\boldsymbol{\Omega}_{\mathbf{1}}, \boldsymbol{\Omega}_{\mathbf{2}}$ and $\boldsymbol{\Omega}_{\mathbf{3}}$ the surfaces :

$$
\begin{aligned}
& \boldsymbol{\Omega}_{\mathbf{1}}=\left\{H_{1}(x, y, z)=0, H_{2}(x, y, z) \geq \frac{-(a-1)^{2}}{4}, H_{2}(x, y, z) \neq 0\right\}, \\
& \boldsymbol{\Omega}_{\mathbf{2}}=\left\{H_{2}(x, y, z)=0, H_{1}(x, y, z) \neq 0\right\}, \\
& \boldsymbol{\Omega}_{\mathbf{3}}=\left\{H_{1}(x, y, z)=c_{1}(t), H_{2}(x, y, z)=c_{2}(t): t \in \mathbb{R} \backslash\{0,1,-a\} \text { and } P(t) \leq 0\right\},
\end{aligned}
$$

where $c_{1}(t)=\frac{(a+2 t-1)^{2}(t+1)^{2}}{t(t-1)(a+t)}$ and $c_{2}(t)=\frac{\left(t-1+t^{2}+a\right)^{3}}{t(a+t)(t-1)^{2}}$.

In our study we omit the case $a=1$ and $a=-1$. The case $a=1$ is the famous Lyness map which is a periodic map of period eight. The case $a=-1$ is omitted because although its behavior is very similar to the general case, the computations are very different.

The result that we prove is the following:

Theorem 13. Let $p \in \mathbb{R}^{3}$ be such that the orbit of $p$ under $F(x, y, z)=\left(y, z, \frac{a+y+z}{x}\right)$ is entirely defined, and assume that $a \neq \pm 1$.

(a) If $p \notin \boldsymbol{\Omega}_{\mathbf{1}} \cup \boldsymbol{\Omega}_{\mathbf{2}} \cup \boldsymbol{\Omega}_{\mathbf{3}}$ then the dynamic of $p$ is standard. 
(b) For $i=1,2,3$, if $p \in \boldsymbol{\Omega}_{\mathbf{i}}$ then the $\alpha$ and the $\omega$ limit of $p$ coincide and it is a periodic orbit contained in $\gamma_{i}$.

We will prove the above result by studying, first, the map $F^{2}$. The reason is because it has a very simple first integral (see [22]), namely,

$$
W(x, y, z)=\frac{(x+1)(z+1)}{y} .
$$

The three functions $H_{1}(x, y, z), H_{2}(x, y, z)$ and $W(x, y, z)$ are first integrals of $F^{2}$ and they are functionally dependent. In fact, a simple computation shows that

$$
(1+W(x, y, z)) H_{1}(x, y, z)=W(x, y, z)\left(a-2+H_{2}(x, y, z)-W(x, y, z)\right) .
$$

For each $k \notin\{0, \infty\}$ the equation $W(x, y, z)=k$ allow us to isolate $y$ getting $y=$ $\frac{(x+1)(z+1)}{k}$. This fact makes possible to work in dimension two. In fact, by substituting the expression of $y$ in $F^{2}(x, y, z)$ and in $H_{1}(x, y, z)$ we are going to study the corresponding dynamic, i. e., the iterations of the two dimensional integrable systems

$$
f_{k}(x, z)=\left(z, \frac{(k+1) z+a k+1}{x(z+1)}\right)
$$

with inverse

$$
g_{k}(x, z)=\left(\frac{(k+1) x+1+a k}{z(x+1)}, x\right)
$$

and with

$$
H_{k}(x, z)=\frac{(1+a k+(k+1) x+(k+1) z+x z)(1+k+x+z+x z)}{k x z}
$$

as a first integral.

The level sets are ${ }^{k} \Sigma_{c}=\left\{(x, z): \psi_{k, c}(x, z)=0\right\}$ where

$$
\psi_{k, c}(x, z)=(1+a k+(k+1) x+(k+1) z+x z)(1+k+x+z+x z)-c k x z .
$$

The other ingredient that we are going to use is the differential equation associated to $f_{k}(x, z)$. A simple computation shows that the function $\mu(x, z)=x z$ satisfies condition (1) and that the corresponding vector field is

$$
X_{k}(x, z)=\left[-\frac{(x+1+k)\left(-k x-a k+z^{2} x-1+z^{2}-x\right)}{k x} \frac{\partial}{\partial x}+\frac{(1+k+z)\left(-z k-a k-1+x^{2}+z x^{2}-z\right)}{k z} \frac{\partial}{\partial z}\right] .
$$

Now, we begin by studying the critical points of the vector field $X_{k}$. Note that since $X_{k}$ has $H_{k}(x, z)$ as a first integral, a critical point of $X_{k}$ is isolated in his level set if and only if it is a center. 
Proposition 14. Set $a \neq \pm 1$ and $k \neq 0, \infty$. Then the following holds

(1) The vector field $X_{k}$ has one, two, or three critical points of the form $(t, t)$ with $t$ satisfying the equation

$$
t^{3}+t^{2}-(1+k) t-(1+a k)=0
$$

These critical points are centers if and only if $P(t)>0$, where

$$
P(t)=(t+1)\left(2 t^{2}+(3 a-1) t+1-a\right)(2 t+a-1)\left(t^{2}+t+a-1\right)^{2} .
$$

Moreover these critical points are fixed points of $f_{k}$ and its level set is ${ }^{k} \Sigma_{c_{1}(t)}$.

(2) If $k \neq-1, a-2$ then $X_{k}$ has also the following critical points:

$$
a_{k}^{1}=(-1-k,-1-k), a_{k}^{2}=\left(-1-k, \frac{a-2-k}{1+k}\right), a_{k}^{3}=\left(\frac{a-2-k}{1+k},-1-k\right) .
$$

The three points are saddles and they form a three periodic orbit of $f_{k}$. Moreover these three critical points belong to the level set ${ }^{k} \Sigma_{c^{*}(k)}$ where $c^{*}(k)=\frac{k(a-2-k)}{k+1}$. When $a \leq \frac{5}{4}$ and $k=\frac{-1 \pm \sqrt{5-4 a}}{2}$ the three critical points collapse to a single critical point which is not a center and it is a fixed point of $f_{k}$.

(2)' If $k=a-2$ then $X_{k}$ has also the critical point $(-1-k,-1-k)$ which is a saddle and it belongs to the level set ${ }^{a-2} \Sigma_{0}$. Moreover $f_{k}$ is not defined at this point.

(3) If $k \geq \frac{-(a-3)^{2}}{4}$ and $k \neq a-2$ then $X_{k}$ has also the following critical points

$$
\begin{aligned}
& b_{k}^{1}=\left(\frac{1-a+\sqrt{a^{2}-6 a+9+4 k}}{2}, \frac{1-a-\sqrt{a^{2}-6 a+9+4 k}}{2}\right), \\
& b_{k}^{2}=\left(\frac{1-a-\sqrt{a^{2}-6 a+9+4 k}}{2}, \frac{1-a+\sqrt{a^{2}-6 a+9+4 k}}{2}\right),
\end{aligned}
$$

which are saddles and form a two periodic orbit of $f_{k}$. Both points belongs to the level set ${ }^{k} \Sigma_{0}$. When $k=\frac{-(a-3)^{2}}{4}$ both critical points collapse to a single critical point which is not a center and it is a fixed point of $f_{k}$.

Proof.

(1) Let $t$ be a solution of equation (17). Easy computations show that $(t, t)$ is a critical point of $X_{k}$ and its level set is $c_{1}(t)$. Then the algebraic curve $H_{k}(x, z)=c_{1}(t)$ writes as

$$
P_{2}(x-t, z-t)+P_{3}(x-t, z-t)+P_{4}(x-t, z-t)=0,
$$

where for $i=1,2,3, P_{i}$ is a homogeneous polynomial of degree $i$ with coefficients rational functions on $a$ and $t$.

A computation shows that $d(t)=-\frac{P(t)(t+1)^{4}}{t^{2}(a+t)^{4}}$ is the discriminant of $P_{2}$. Thus if $P(t)>0$ then the point $(t, t)$ is isolated in its level set and therefore it is a center of the vector field 
$X_{k}$. Reciprocally, if $P(t)<0$ then the point $(t, t)$ has two real straight lines tangent to the level set, so the point $(t, t)$ is a saddle. Hence it only remains to prove that when $P(t)=0$ the critical point $(t, t)$ is not a center (that is, it is not isolated in its level set). So assume that $P(t)=0$. This can be done in four different situations:

(a) $t^{2}+t+a-1=0$. This implies that $P_{2}$ is identically 0 and the level curve begins with third order terms. So it has at least one real tangent at $(t, t)$ and this point is not a center.

(b) $t=\frac{1-a}{2}$. This implies that when $a \neq 3, P_{2}(x, z)=A((x-t)+(z-t))^{2}$ with $A \neq 0$ which implies that the point $(t, t)$ is not a center in this case. When $a=3, P_{2}$ and $P_{3}$ are identically 0 and the level curve writes as $B(x-t)^{2}(z-t)^{2}, B \neq 0$ which has two double tangents.

(c) $2 t^{2}+(3 a-1) t+1-a=0$. This implies that $P_{2}(x, z)=C((x-t)-(z-t))^{2}$ with $C \neq 0$ which also implies that the point is not a center.

(d) $t=-1$. This implies $k=0$ or $a=1$ which are not under our hypotheses.

Statements (2), (2)' and (3) follow in a very similar way. We omit the details for shortness.

Now for each $k \neq 0, \infty$ we define the finite set $\bar{c}(k) \subset \mathbb{R}$ as follows. We say that $c_{0} \in \bar{c}(k)$ if and only if $c_{0}=c_{1}(t)$ for some $t \in \mathbb{R}$ verifying $k=k(t)$ and $P(t) \leq 0$. Notice that $\bar{c}(k)$ can be empty and it has at most three elements. Also set $c^{*}(k)=\frac{k(a-2-k)}{k+1}$. From the above lemma the only level sets ${ }^{k} \Sigma_{c}$ containing critical points of the differential equation are when $c \in \bar{c}(k) \cup\left\{0, c^{*}(k)\right\}$. Notice that ${ }^{k} \Sigma_{c^{*}(k)},{ }^{k} \Sigma_{0}$ (when $k \geq-\frac{(a-1)^{3}}{2}$ ) and ${ }^{k} \Sigma_{c}$ when $c \in \bar{c}(k)$ contain the periodic orbits

$$
\begin{gathered}
O_{c^{*}(k)}^{k}=\left\{(-1-k,-1-k),\left(-1-k, \frac{a-2-k}{k+1}\right),\left(\frac{a-2-k}{k+1},-1-k\right)\right\}, \\
O_{0}^{k}=\left\{\left(\frac{1-a \pm \sqrt{(a-3)^{2}+4 k}}{2}, \frac{1-a \mp \sqrt{(a-3)^{2}+4 k}}{2}\right)\right\},
\end{gathered}
$$

and

$$
O_{\bar{c}(k)}^{k}=\left\{(t, t): t^{3}+t^{2}-(k+1) t-(1+a k)=0 \text { and } P(t) \leq 0\right\},
$$

respectively.

The other main ingredient to describe the dynamics of $f_{k}$ is the study of the points added to compactify the level sets.

As in the previous example we introduce the following points: 


$$
\begin{array}{rrrr}
P_{1}=(-k-1, \infty) & , \quad P_{2}=(\infty,-1) \quad, \quad P_{3}=(-1, \infty) \quad, \quad P_{4}=(\infty,-k-1), \\
Q_{1}=(-k-1,-\infty) & , \quad Q_{2}=(-\infty,-1) \quad, \quad Q_{3}=(-1,-\infty) \quad, \quad Q_{4}=(-\infty,-k-1),
\end{array}
$$

and

$$
\begin{gathered}
\mathbf{x}=(0,-k-1) \quad, \quad \mathbf{y}=\left(0,-\frac{1+a k}{k+1}\right) \quad, \quad \mathbf{z}_{\mathbf{c}}=\left(\frac{(1-a) k}{k+c},-1\right), \\
\mathbf{t}=(-k-1,0) \quad, \quad \mathbf{v}_{\mathbf{c}}=\left(-1, \frac{(1-a) k}{k+c}\right) .
\end{gathered}
$$

Notice that when $k=-1$, the point $\mathbf{y}$ disappears, when $k=a-2$ the points $\mathbf{x}$ and $\mathbf{y}$ coincide and when $c=-k$, the points $\mathbf{z}_{\mathbf{c}}, \mathbf{v}_{\mathbf{c}}$ disappear. Note also that the points $\mathbf{x}, \mathbf{y}$ and $\mathbf{t}$ belong to all the level sets while the points $\mathbf{z}_{\mathbf{c}}, \mathbf{v}_{\mathbf{c}}$ belong to the level ${ }^{k} \Sigma_{c}$.

Lemma 15. For each $k \notin\{0, \infty\}$ the following schemes work:

(a) For $c \notin\{-k, \infty\}$

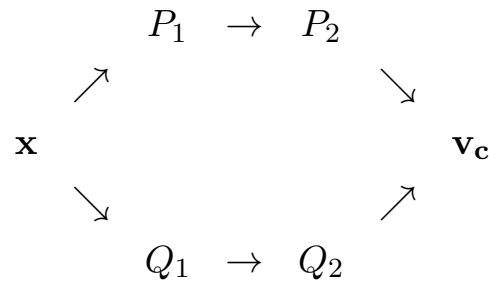

and

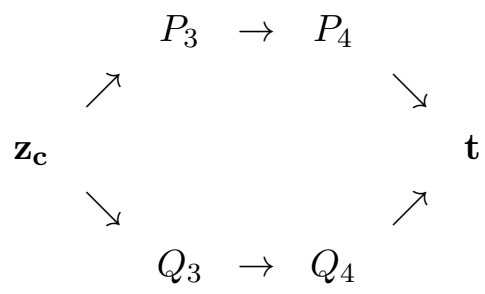

(b) For $c=-k$ the points $\mathbf{z}_{\mathbf{c}}$ and $\mathbf{v}_{\mathbf{c}}$ disappear, and

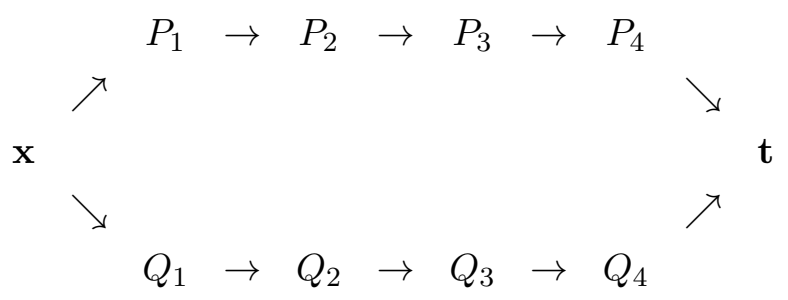

(c) If $k \neq a-2$ the extended map $f_{k}$ on ${ }^{k} \Sigma_{c}$ satisfies

$$
f_{k}(\mathbf{y})=\frac{k(k-a+2)^{2}+c(1+k)(k a+1)}{k(a-1)(k-a+2)} .
$$


Proof. It is and easy computation.

Proposition 16. For each $k \notin\{0, \infty\}$ consider the map (14)and set $p \in \mathbb{R}^{2}$. Then the following statements hold:

(a) If $k \notin\{a-2,-1\}$ and $k \geq \frac{-(a-3)^{2}}{4}$ then

(a) If $p \notin^{k} \Sigma_{0} \cup^{k} \Sigma_{c^{*}(k)} \cup\left(\cup_{c \in \bar{c}(k)}{ }^{k} \Sigma_{c}\right)$ then the dynamic of $p$ is standard.

$\left(a_{2}\right)$ If $p \in{ }^{k} \Sigma_{0}$ then $\alpha(p)=\omega(p)=O_{0}^{k}$.

(a) If $p \in{ }^{k} \Sigma_{c^{*}(k)}$ then $\alpha(p)=\omega(p)=O_{c^{*}(k)}^{k}$.

$\left(a_{4}\right)$ If $p \in\left(\cup_{c \in \bar{c}(k)}{ }^{k} \Sigma_{c}\right)$ then the $\alpha$ and the $\omega$ limit of $p$ coincide and it is a fixed point contained in $O_{\bar{c}(k)}^{k}$.

(b) If $k \notin\{a-2,-1\}$ and $k<\frac{-(a-3)^{2}}{4}$ then:

$\left(b_{1}\right)$ If $p \notin{ }^{k} \Sigma_{c^{*}(k)} \cup\left(\cup_{c \in \bar{c}(k)}{ }^{k} \Sigma_{c}\right)$ then the dynamic of $p$ is standard.

$\left(b_{2}\right)$ If $p \in{ }^{k} \Sigma_{c^{*}(k)}$ then $\alpha(p)=\omega(p)=O_{c^{*}(k)}^{k}$.

$\left(b_{3}\right)$ If $p \in\left(\cup_{c \in \bar{c}(k)}{ }^{k} \Sigma_{c}\right)$ then the $\alpha$ and the $\omega$ limit of $p$ coincide and it is a fixed point contained in $O_{\bar{c}(k)}^{k}$.

(c) If $k=a-2$ then $c^{*}(a-2)=0$ and

$\left(c_{1}\right)$ If $p \notin^{a-2} \Sigma_{0} \cup\left(\cup_{c \in \bar{c}(a-2)}{ }^{a-2} \Sigma_{c}\right)$ then the dynamic of $p$ is standard.

$\left(c_{2}\right)$ If $p \in{ }^{a-2} \Sigma_{0}$ then the orbit of $p$ is not entirely defined.

$\left(c_{3}\right)$ If $p \in\left(\cup_{c \in \bar{c}(a-2)}{ }^{a-2} \Sigma_{c}\right)$ then the $\alpha$ and the $\omega$ limit of $p$ coincide and it is a fixed point contained in $O_{\bar{c}(a-2)}^{a-2}$.

(d) If $k=-1$ then $c^{*}(-1)=0$ and

$\left(d_{1}\right)$ If $p \notin^{-1} \Sigma_{0} \cup\left(\cup_{c \in \bar{c}(-1)}{ }^{-1} \Sigma_{c}\right)$ then the dynamic of $p$ is standard.

$\left(d_{2}\right)$ If $p \in{ }^{-1} \Sigma_{0}$ and $a \notin(1,5)$ then $\alpha(p)=\omega(p)=O_{0}^{-1}$.

$\left(d_{2}\right)^{\prime}$ If $p \in{ }^{-1} \Sigma_{0}$ and $a \in(1,5)$ then the dynamic of $p$ is standard.

$\left(d_{3}\right)$ If $p \in\left(\cup_{c \in \bar{c}(-1)}{ }^{-1} \Sigma_{c}\right)$ then the $\alpha$ and the $\omega$ limit of $p$ coincide and it is a fixed point contained in $O_{\bar{c}(-1)}^{-1}$.

Proof. It is a direct application of Theorem B taking into account that from Lemma 15 it follows that for any $k$ and any $c \notin \bar{c}(k) \cup\left\{0, c^{*}(k)\right\}$, the set ${ }^{k} E_{c}$ has not invariant subsets. On the other hand for $c \in \bar{c}(k) \cup\left\{0, c^{*}(k)\right\}$, the invariant subsets contained in ${ }^{k} E_{c}$ are described in Proposition 14. 
We remark that the level ${ }^{a-2} \Sigma_{0}$ is not regular because there is a straight line contained in the level on which the determinant of the differential of $f_{a-2}$ is identically 0 . Then this level is not under the hypotheses of the Theorem A. A simple computation shows that either $f_{a-2}(x, z)=(0, a-1)$ or $f_{a-2}^{2}(x, z)=(0, a-1)$ for any $(x, z) \in{ }^{a-2} \Sigma_{0}$, so $f_{a-2}^{3}$ is not defined at any point in the level.

Proof of Proposition 13. For $p \in \mathbb{R}^{3}$ denote by $c=H_{1}(p), k=W(p)$ and $c_{2}=H_{2}(p)$. Then from (13) we get the relation

$$
(1+k) c=k\left(c_{2}+a-2-k\right)
$$

We begin by proving statement $(b)$. Take $p \in \boldsymbol{\Omega}_{\mathbf{1}}$, i.e., assume that $c=0$ and $c_{2} \geq$ $-\frac{(a-1)^{2}}{4}$ with $c_{2} \neq 0$. Then from (19) either, $c=0$ and $k \geq-\frac{(a-3)^{2}}{4}$ with $k \notin\{0, a-2\}$ or $c=0, k=0$.

In the first case we consider the projection of $p$ in the plane $(x, z)$ and we apply statement $\left(a_{2}\right)$ of Proposition 16. Notice that $p=\left(x, \frac{(x+1)(z+1)}{k}, z\right)$, so for $k \notin\{0, a-2\}$ and $k \geq$ $-\frac{(a-3)^{2}}{4}$ the set

$$
\tilde{O}_{0}^{k}=\left\{\left(\frac{1-a \pm \sqrt{(a-3)^{2}+4 k}}{2},-1, \frac{1-a \mp \sqrt{(a-3)^{2}+4 k}}{2}\right)\right\}
$$

form a 2-periodic orbit of $F^{2}(x, y, z)$ in $\mathbb{R}^{3}$. So, if we consider

$$
F\left(\tilde{O}_{0}^{k}\right)=\left\{\left(-1, \frac{1-a \mp \sqrt{(a-3)^{2}+4 k}}{2},-1\right)\right\}
$$

we get that $\tilde{O}_{0} \cup F\left(\tilde{O}_{0}\right)$ is a 4-periodic orbit of $F(x, y, z)$ in $\mathbb{R}^{3}$ and it is clearly contained in $\gamma_{1}$. So, if the orbit of $p$ is entirely defined, its alpha and omega limit are contained in $\gamma_{1}$ as we wanted to prove.

In the second case consider a point $p$ satisfying $c=0$ and $k=0$. Then either, $W(F(p))=$ 0 or $W(F(p)) \neq 0$. If $W(F(p)) \neq 0$, then we argue as in the previous paragraph taking the point $q=F(p)$ and the result follows.

Now assume that $W(p)=W(F(p))=0$, i.e., $(x+1)(z+1)=0$ and $(y+1)(a+x+y+z)=$ 0 . Since through $F$ we get:

$$
\begin{aligned}
& \{x+1=0, y+1=0\} \rightarrow\{x+1=0, a+x+y+z=0\} \rightarrow\{z+1=0, a+x+y+z=0\} \\
& \rightarrow\{y+1=0, z+1=0\} \rightarrow\{x+1=0, y+1=0\}
\end{aligned}
$$

we are going to take into account the map $F^{4}(x, y, z)$. Notice that the restriction of $F^{4}$ from $(x+1)(z+1)=0$ to itself reduces to $(-1-1, z) \rightarrow(-1,-1, \varphi(z))$ where $\varphi(z)=\frac{2-a}{a-1+z}$. The dynamics of $z \rightarrow \frac{2-a}{a-1+z}$ is known (see [6], for instance). This map has two fixed points (resp., one fixed point), $z=-1, z=2-a$ (resp. $z=-1$ ) for $k \neq a-3$ (resp. $k=a-3$ ). 
For $a \neq 2$, these two points are one attractor and the other one repellor (resp. attractor). And all the other points have this fixed points in the alpha and omega limit set. Since through $F$ :

$$
(-1,-1,-1) \rightarrow(1,-1,2-a) \rightarrow(-1,2-a,-1) \rightarrow(2-a,-1-1) \rightarrow(-1,-1,-1)
$$

and this periodic set is contained in $\gamma_{1}$, the result follows and $(b)$ is proved.

Now let $p \in \boldsymbol{\Omega}_{2}$, i.e., assume that $H_{2}(p)=0, H_{1}(p) \neq 0$. Int his case equation (19) writes as

$$
(1+k) c=k(a-2-k) .
$$

Hence, $c \neq 0, k \notin\{0, a-2,-1\}$ and $c=c^{*}(k)$. As before we consider the following periodic sets:

$$
\tilde{O}_{c^{*}(k)}^{k}=\left\{(-1-k, k,-1-k),\left(-1-k, \frac{1-a}{k+1}, \frac{a-2-k}{k+1}\right),\left(\frac{a-2-k}{k+1}, \frac{1-a}{k+1},-1-k\right)\right\}
$$

and

$$
F\left(\tilde{O}_{c^{*}(k)}^{k}\right)=\left\{\left(k,-1-k, \frac{1-a}{k+1}\right),\left(\frac{1-a}{k+1}, \frac{a-2-k}{k+1}, \frac{1-a}{k+1},\right),\left(\frac{1-a}{k+1},-1-k, k\right)\right\} .
$$

The set $\tilde{O}_{c^{*}} \cup F\left(\tilde{O}_{c^{*}}\right)$ form a 6 -periodic orbit of $F$ in $\mathbb{R}^{3}$ and it is contained in $\gamma_{2}$. Now applying $\left(a_{3}\right)$ and $\left(b_{2}\right)$ of Proposition 16 the result follows.

Lastly assume that $p \in \boldsymbol{\Omega}_{3}$. That is $H_{1}(p)=c_{1}(t), H_{2}(p)=c_{2}(t)$ for some $t \in \mathbb{R} \backslash$ $\{0,1,-a\}$ with $P(t) \leq 0$. Since the 2-periodic point $v=\left(t, \frac{a+t}{t-1}, t\right)$ verifies that $H_{1}(v)=c_{1}(t)$ and $H_{2}(v)=c_{2}(t)$, it follows that either $W(p)=W(v)$ or $W(F(p))=W(v)$. Assume for instance that $W(p)=W(v)=k$. Then it follows that $c_{1}(t) \in \tilde{c}(k)$ and the result follows from the statements (a4),(b3),(c3) and (d3) of Proposition 16. Since the omega limit and alfa limit of $p$ and $F(p)$ coincide the result follows also in the other case.

In order to prove $(a)$ consider a point $p \notin \boldsymbol{\Omega}_{1} \cup \boldsymbol{\Omega}_{2} \cup \boldsymbol{\Omega}_{3}$. First of all note that since $p \notin \boldsymbol{\Omega}_{3}$ then $c \notin \tilde{c}(k)$. Hence we have four cases: $c=\infty, c=0=c_{2}, c=0$ with $c_{2}<-\frac{(a-1)^{2}}{4}$ and $c \neq 0 \neq c_{2}$.

Clearly when $c=\infty$ the orbit of $p$ is not entirely defined. Also as we noticed in Example 1, when $H_{1}(p)$ and $H_{2}(p)$ are zero the orbit of $p$ is not defined. On the other hand condition $c_{2} \neq 0$ implies that $c \neq c^{*}(k)$. Otherwise, since $c^{*}(k)=\frac{k(a-2-k)}{k+1}$ we have that $(k+1) c^{*}(k)=k(a-2-k)$ and from $(13)$ we get $c_{2}=0$.

Now assume that $c=0$ and $c_{2}<-\frac{(a-1)^{2}}{4}$ with $c_{2} \neq 0$. Then $k<-\frac{(a-3)^{2}}{4}$ and since $c \neq c^{*}(k)$ the result follows from $\left(b_{1}\right)$ of Proposition 16 .

Finally if $c \neq 0 \neq c_{2}$ then if $k \notin\{a-2,-1\}$ since $c \neq c^{*}(k)$ the result follows from $\left(a_{1}\right)$ and $\left(b_{1}\right)$ of Proposition 16. If $k=a-2$ (resp. $k=-1$ ) we apply $\left(c_{1}\right)$ (resp. $\left(d_{1}\right)$ ). 


\section{References}

[1] G. Bastien and M. Rogalski. Global Behavior of the Solutions of Lyness Difference equation $u_{n+2} u_{n}=u_{n+1}+a$, J. Difference Equations and Appl. 10, no. 11 (2004), 977-1003.

[2] G. Bastien and M. Rogalski. On algebraic difference equations $u_{n+2}+u_{n}=\psi\left(u_{n+1}\right)$ in $\mathbb{R}$ Related to a Family of Elliptic Quartics in the Plane, J. Math. Anal. Appl. 326 (2007), 822-844.

[3] Bochnak, J., Coste, M. and Roy, M-F Real algebraic geometry., Springer-Verlag, Berlin, (1998)

[4] A. Cima, A. Gasull, V. Mañosa. Global periodicity and complete integrability of discrete dynamical systems, J. Difference Equ. Appl. 12 (2006), 697-716.

[5] A. Cima, A. Gasull, V. Mañosa. Dynamics of the third order Lyness' difference Equation, to appear in J. Difference Equ. Appl.

[6] A. Cima, A. Gasull, V. Mañosa. Studying discrete dynamical systems via differential equations, J. Differential Equations, 244 (2008), 630-648.

[7] A. Cima, A. Gasull, V. Mañosa. Some properties of the $k$-dimensional Lyness map, J. Physics A, 41(2008), 1-18.

[8] A. Cima, A. Gasull, V. Mañosa. On Poncelet's Maps, To appear.

[9] J. Esch,T. D. Rogers. The Screenaver Map: Dynamics on Elliptic Curves Arising from Polygonal Folding, Discrete Comput Geom, 25(2001), 477-502.

[10] L. Gardini, G.I. Bischi, C. Mira. Invariant curves and focal points in a Lyness iterative process, Internat. J. Bifur. Chaos Appl. Sci. Engrg. 13 (2001), 1841-1852.

[11] M. Gao, Y Kato, M. Ito. Some Invariants for $k^{\text {th }}$-Order Lyness Equation Applied Mathematics Letters 17 (2004) 1183-1189.

[12] I. Gumovski, Ch. Mira. "Recurrences and discrete dynamic systems". Lecture Notes in Mathematics 809. Springer Verlag, Berlin, 1980.

[13] F.A. Haggar, G.B. Byrnes, G.R. Quispel and H.W. Cappel. k-integrals and k-Lie symmetries in discrete dynamical systems Phys. A 233 (1996) 379-394.

[14] R. Hirota, K. Kimura and H. Yahagi. How to find the conserved quantities of nonlinear discrete equations J. Phys. A: Math. Gen. 34 (2001) 10377-10386. 
[15] A. Iatrou. Three dimensional integrable mappings arXiv:nlin.SI/0306052vl (2003)

[16] A. Iatrou. Higher dimensional integrable mappings Phys. D: 179 (2003) 229-253.

[17] A. Iatrou and , J.A.G. Roberts. Integrable mappings of the plane preserving biquadratic invariant curves J. Phys. A: Math. Gen. 34 (2001) 6617-6636.

[18] A. Iatrou and, J.A.G. Roberts. Integrable mappings of the plane preserving biquadratic invariant curves II Nonlinearity 15 (2002) 459-489.

[19] A. Iatrou and , J.A.G. Roberts. Integrable mappings of the plane preserving biquadratic invariant curves III Phys. A 326 (2003), no. 3-4, 400-411.

[20] D. Jogia, J.A.G. Roberts and F. Vivaldi. An algebraic geometric approach to integrable maps of the plane Phys. A 39 (2006), 1133-1149.

[21] S. Lafortune, A. Goriely. Singularity confinement and algebraic integrability J. Math. Physics 45, N3 (2004) 1191-1208.

[22] J. Matsukidaira, D. Takahashi. Third-order integrable difference equations generated by a pair of second-order equations, J. Phys. A. Math Gen. 39 (2006), 1151-1161.

[23] J.A.G. Roberts, G.R.W. Quispel. Creating and relating three-dimensional integrable maps, J. Phys. A. Math Gen. 39 (2006), L605-L615.

[24] T. Tsuda. Integrable mappings via rational elliptic surfaces, J. Phys. A. Math Gen. 37 (2004), 2721-2730.

[25] P. Walters. An introduction to eergodic theory, Springer (1992).

[26] E. C. Zeeman. Geometric unfulding of a difference equation, Unpublished paper. Hertford College, Oxford(1996), 1-42. 